\title{
Structural insights into the BRAF monomer-to-dimer transition mediated by RAS binding
}

\author{
Juliana A. Martinez Fiesco ${ }^{1 \wedge}$, David E. Durrant ${ }^{2 \wedge}$, Deborah K. Morrison ${ }^{2 *}$ \\ and Ping Zhang ${ }^{*}$ \\ ${ }^{1}$ Center for Structural Biology and ${ }^{2}$ Laboratory of Cell and Developmental Signaling, Center for \\ Cancer Research, National Cancer Institute-Frederick, Frederick, MD 21702
}

$\wedge$ These authors contributed equally to this work

* corresponding authors: morrisod@mail.nih.gov and ping.zhang@nih.gov 


\begin{abstract}
RAF kinases are essential effectors of RAS, but how RAS binding initiates the conformational changes needed for autoinhibited RAF monomers to form active dimers has remained unclear. Here, we present cryo-electron microscopy structures of full-length BRAF complexes derived from mammalian cells: autoinhibited, monomeric BRAF:14-3-32:MEK and BRAF:14-3-32 complexes, and an inhibitor-bound, dimeric $\mathrm{BRAF}_{2}: 14-3-3_{2}$ complex, at 3.7, 4.1, and $3.9 \AA$ resolution, respectively. In both autoinhibited, monomeric structures, the RAS binding domain (RBD) of BRAF is resolved, revealing that the RBD forms an extensive contact interface with the 14-3-3 protomer bound to the BRAF C-terminal site and that key basic residues required for RBDRAS binding are exposed. Moreover, through structure-guided mutational studies, our findings indicate that RAS-RAF binding is a dynamic process and that RBD residues at the center of the RBD:14-3-3 interface have a dual function, first contributing to RAF autoinhibition and then to the full spectrum of RAS-RBD interactions.
\end{abstract}




\section{Introduction}

The RAF kinases (ARAF, BRAF and CRAF/RAF1) are key intermediates in the RAS pathway, functioning in the transmission of signals that regulate cell proliferation, differentiation, and survival $^{1}$. Over the years, biochemical studies have provided critical insights regarding the mechanisms that regulate RAF signal output ${ }^{2,3}$. In quiescent cells, members of the RAF kinase family localize to the cytosol as inactive monomers ${ }^{4}$, maintained in an autoinhibited state through intramolecular interactions between the RAF regulatory and catalytic domains and by the binding of a 14-3-3 dimer to two phosphorylation-dependent serine sites (pS365 and pS729 in BRAF) ${ }^{5-8}$. In response to signaling events and RAS activation, RAF interacts directly with GTP-bound RAS at the plasma membrane ${ }^{9-12}$, which ultimately disrupts the autoinhibited state and promotes RAF dimerization and kinase activation ${ }^{13-16}$.

Numerous structures of dimerized RAF kinase domains and other isolated regions of RAF have been solved using X-ray crystallography or NMR spectroscopy; however, the structural determination of full-length monomeric and dimeric RAF complexes has been challenging due to the number of proteins that associate with RAF as well as the largely unstructured nature of the Nterminal regulatory domain. Nevertheless, recent cryo-electron microscopy (cryo-EM) and cry stal structures of BRAF have shed light on these complexes, providing visual context to the knowledge obtained through biochemical and cell biological approaches ${ }^{17-20}$. For example, the published cryo-EM structure of an autoinhibited, monomeric BRAF complex confirmed that a 14-3-3 dimer can bind simultaneously to the BRAF pS365 and pS729 sites and that the CRD makes critical contacts with both the RAF catalytic domain and the 14-3-3 dimer in the autoinhibited state ${ }^{17}$, consistent with previous studies implicating the CRD and 14-3-3 as key regulators of RAF autoinhibition ${ }^{5-8}$. Binding of the 14-3-3 dimer was also found to occlude both the membrane/ligand binding region of the $\mathrm{CRD}$ and the dimer interface of the kinase domain ${ }^{17}$, thus preventing spurious dimer formation and CRD-membrane contact. In contrast, the RBD was not sufficiently resolved in the autoinhibited complex to determine its exact position or orientation ${ }^{17}$. As a result, questions regarding whether the RBD interacts with other BRAF domains or the 14- 
3-3 dimer in the autoinhibited state remained open. Moreover, no insight was gained regarding how RAS binding relieves RAF autoinhibition and promotes the conformational changes required for dimerization and kinase activation. In addition, recent BRAF dimer structures have shown that the 14-3-3 dimer can bind simultaneously to two kinase domain protomers, but whether both protomers are catalytically active has been a matter of debate ${ }^{17-20}$.

Using a mammalian cell expression system to isolate BRAF complexes, here we report the cryo-EM structures of two autoinhibited, monomeric BRAF complexes in which the RBD is welldefined. Only one of the autoinhibited complexes included MEK, but both contained a bound 143-3 dimer, and in both structures, the kinase domain was in a canonical inactive configuration and lacked ATP. In addition, we also report the cryo-EM structure of a BRAF dimer complex with both BRAF protomers bound to an ATP-competitive BRAF inhibitor and the kinase domains assuming the active conformation. The dimeric BRAF complex also included a dimer of 14-3-3 that bridged the C-terminal pS729 sites on each BRAF protomer, and the dimerized kinase domains exhibited an asymmetric orientation with respect to the 14-3-3 dimer. Notably, resolution of the RBD in our autoinhibited, monomeric BRAF structures provides key insights regarding how the orientation of the RBD allows access for RAS binding and how the interaction with RAS initiates the monomer-to-dimer transition required for RAF activation.

\section{Results}

\section{Isolation and initial characterization of BRAF complexes}

To isolate BRAF complexes for cryo-EM analysis that would accurately reflect the regulatory mechanisms that take place in mammalian cells, we first established a human 293 FT cell line that stably expressed full-length, wild-type (WT) BRAF containing an N-terminal Halo tag (Fig. 1a). Using this cell line, monomeric BRAF complexes were isolated from quiescent, serum-depleted cells. Dimeric BRAF complexes were also isolated using cycling cells that were treated with the type I BRAF inhibitor SB590885 to promote RAF dimerization. BRAF complexes were collected from cell lysates using affinity chromatography and then separated by gel filtration 
chromatography to obtain homogenous samples containing recombinant BRAF in association with various endogenous proteins (Supplementary Fig. 1a-c). Analysis of the gel filtration fractions obtained from serum-depleted cells indicated a well-defined peak of monomeric BRAF that was comprised of two distinct physiological complexes, a larger complex containing 14-3-3 proteins and MEK (fraction 18) and a smaller complex containing 14-3-3 proteins but lacking MEK (fraction 19) (Supplementary Fig. 1a). To maximize purity and homogeneity of the MEK-bound complex, an additional protein preparation was generated from serum-depleted cells that had been treated with the MEK inhibitor CH5126766 to stabilize the BRAF-MEK interaction (Supplementary Fig. 1b). Analysis of the gel filtration fractions obtained from the RAF inhibitor SB590885-treated cells indicated a prominent peak near fraction 16 that represented a larger complex containing primarily BRAF and 14-3-3 proteins, with MEK and Hsp70 also observed (Supplementary Fig. 1c).

Mass spectrometry analysis of each of the three sample complexes revealed that $14-3-3 \varepsilon$ and $14-3-3 \zeta$ constituted approximately $50 \%$ and $25 \%$, respectively of the $14-3-3$ proteins present in both the monomeric and dimeric BRAF complexes (Supplementary Fig. 1d), a finding consistent with the expression level of these 14-3-3 isoforms in 293FT cells ${ }^{21}$ and the propensity for $14-3-3 \varepsilon$ to heterodimerize 22 . For the MEK-bound monomeric BRAF complex, MEK1 and MEK2 were present at a 54\% and 46\% ratio, respectively (Supplementary Fig. 1d). It should be noted that for the structures derived from cryo-EM, we used the human 14-3-3 $\zeta$ isoform (PDB ID: 4FJ3) as a starting model for the 14-3-3 dimer and MEK1 (PBD ID: 3WGI) to represent MEK in the MEK-bound, autoinhibited complex.

\section{Structural analysis of the inhibitor-bound dimeric $\mathrm{BRAF}_{2}: 14-3-3_{2}$ complex}

A major advance in understanding RAF activation originates from studies showing that under most signaling conditions, dimerization of the kinase domains is required ${ }^{23}$. Many structures of isolated, dimeric BRAF kinase domains have been solved; however, recent structures of BRAF dimer complexes containing 14-3-3 proteins ${ }^{17,18,20}$ have raised new questions regarding the mechanisms 
of dimer activation and whether both protomers in the dimer have catalytic activity. To further investigate these issues, we isolated BRAF dimer complexes from mammalian cells treated with the type 1 BRAF inhibitor SB590885 and obtained a cryo-EM structure of full-length, inhibitorbound $\mathrm{BRAF}_{2}: 14-3-3_{2}$ at 3.9 ̊ resolution (Fig. 1b-c, and Supplementary Fig. 2a-c). It should be noted that despite the presence of MEK in the cryo-EM sample fraction, no density corresponding to MEK was observed in the 3D reconstructions. Additionally, the RBD and CRD as well as other regions of the $\mathrm{N}$-terminal regulatory domain (residues 1-448) were also missing from the density map and are expected to be solvent exposed and flexible. In our inhibitor-bound $\mathrm{BRAF}_{2}: 14-3-3_{2}$ structure, the BRAF kinase domains (KD) adopt a canonical back-to-back orientation with the $\alpha \mathrm{C}$ helices in the active "in" position and the regulatory (R) spines aligned ${ }^{16,24}$ (Fig. 1 b-c, and Supplementary Fig. 2d-e). As expected, a 14-3-3 dimer was bound to each C-terminal pS729 site, forming a stabilizing bridge between the KD protomers.

The orientation of the KD dimer with respect to the 14-3-3 dimer was asymmetric and similar to that previously described for a cryo-EM structure of a ligand-free $\mathrm{BRAF}_{2}: 14-3-3_{2}$ complex $^{18}$ (Fig. 1c), with our overall structure superimposing well onto the ligand-free dimer structure ( $\mathrm{C} \alpha$ root mean square deviation (RMSD) of $1.54 \AA$ for the $\mathrm{BRAF}_{2}: 14-3-3_{2}$ regions). Notably, a major difference between the two structures is that for the unliganded BRAF $2: 14-3-3_{2}$ structure, the distal C-tail segment (F743 to A749) of one KD protomer was found to insert into the active site of the other protomer, thus inhibiting its cataly tic activity. Moreover, insertion of the C-tail segment into the active site was proposed to allow the "inhibited" protomer to act as a transactivator of the promoter from which the C-tail emanated and was also thought to account for the asymmetric orientation of the KD and 14-3-3 dimers.

In our inhibitor-bound $\mathrm{BRAF}_{2}: 14-3-3_{2}$ structure, interpretable density for both C-tails stops at residue 733, indicating that the C-tails (residues 734 to 766) are flexible after exiting the 14-33 binding pocket (Fig. 1b-c). In addition, density corresponding to the RAF inhibitor SB590885 was observed in both active sites (Fig. 1b-c, and Supplementary Fig. 2d) and overlapped with the position of the $\mathrm{C}$-tail segment in the ligand-free dimer structure (Fig. 1c). This finding shows that 
insertion of the C-tail into the active site can be overcome by inhibitor binding and is consistent with recent biochemical analyses of active $\mathrm{BRAF}_{2}: 14-3-3_{2}$ complexes using differential scanning fluorimetry, microscale thermophoresis, and surface plasmon resonance approaches, which indicated that both ATP binding sites are available for ligand binding even when the C-tail segment is present ${ }^{19}$. In addition, alignment of our inhibitor (SB590885)-bound $\mathrm{BRAF}_{2}: 14-3-3_{2}$ structure with the high resolution crystal structure of inhibitor (GDC-0879)-bound BRAF KD (residues D432-R735) $2: 14-3-3_{2}$ complexes $^{20}$ yielded a Ca RMSD of $2.29 \AA$ for the overall complex, with the main area of difference being one 14-3-3 protomer that is tilted closer to the BRAF kinase domain in the crystal structure, which may be due to the constraints of the crystallographic lattice (Supplementary Fig. 2f). For the GDC-0879-bound structure, inhibitor was also present in both active sites, and even though the KDs lacked the C-tail segments, an asymmetric orientation between the BRAF KD and the 14-3-3 dimer was observed. Taken together, our findings add support to the model that the asymmetry between the KD and 14-3-3 dimers is not determined by C-tail insertion and that the active sites of both protomers may bind ATP simultaneously to promote catalysis.

\section{Structural analysis of the monomeric BRAF:14-3-32:MEK and BRAF:14-3-3 complexes}

Over the years, less has been known regarding the structure of autoinhibited RAF monomers, with only one cryo-EM structure reported for full-length monomeric BRAF 17 . Here, using serumdepleted mammalian cells, we were able to isolate two monomeric BRAF complexes of sufficient homogeneity for structural analysis using cryo-EM, resulting in a BRAF:14-3-32:MEK structure of $3.7 \AA$ resolution and a BRAF:14-3-32 structure of $4.1 \AA$ resolution (Fig. 2a-b, and Supplementary Fig. 3a-c). Except for the presence or absence of MEK, the overall conformation of the monomeric BRAF structures aligned well, with an Ca RMSD of $0.95 \AA$ for the corresponding BRAF: 14-3-32 portions and a C $\alpha$ RMSD of $0.99 \AA$ for the BRAF KDs. In these structures, the BRAF monomer exhibits an autoinhibited conformation with a 14-3-3 dimer bound to the canonical pS365 and pS729 sites and the CRD making stabilizing contacts with the C-lobe 
of the kinase domain and with both protomers of the 14-3-3 dimer (Fig. 2a-b, and Supplementary Fig. 4). These results are consistent with the previously determined BRAF:14-3$3_{2}:$ MEK 1 S218A,S222A structure ${ }^{17}$; however, they show that BRAF can exist in the autoinhibited state in the absence of MEK. Moreover, our structures suggest that binding of the 14-3-3 dimer and intramolecular interactions are sufficient to maintain the autoinhibited conformation, but that MEK binding may further stabilize the complex, as evidenced in the local resolution maps (Supplementary Fig. 3a-b).

The BRAF kinase domain in both monomer structures displayed the canonical kinase inactive conformation ${ }^{16,25}$, with the $\alpha \mathrm{C}$-helix in the "out" position and the R-spine broken (Fig. 2a and Supplementary Fig. 5a, and Supplementary Tables 1-2). Residues in the activation segment (residues 598-602) formed an inhibitory turn to reinforce the extended outward shift of the $\alpha \mathrm{C}$ helix (Supplementary Fig. 5a). Although the ATP binding pocket was unoccupied in our monomer structures, the structure of the KDs displayed a similar conformation as did recent inactive KD structures bound to ATP analogs, namely the ATP- $\gamma-$ S-bound BRAF:14-3-32:MEK1 S218A,S222A structure $^{17}$ (C $\alpha$ RMSD of $0.90 \AA$ for the KDs) and the BRAF KD (AMP-PCP):MEK1 structure ${ }^{20}$ (C $\alpha$ RMSD of $0.82 \AA$ for the KDs) (Supplementary Fig. 5b-c, and Supplementary Tables 1-2). Moreover, as was reported for the ATP-analog-bound structures, the $\mathrm{N}$ - and C-lobes of the KD in our ATP-free structure exhibited a closer orientation than is observed for the lobes of KDs bound to RAF inhibitors (Supplementary Fig. 5b, and Supplementary Table 2) 17,20,26,27. These findings indicate that the ATP binding pocket of the BRAF KD is stable in the "apo" nucleotide-free state and that while ATP-binding may be required to form the compact configuration of the N-and Clobes as well as the autoinhibited BRAF conformation, these states can exist in the absence of bound ATP (Supplementary Fig. 5b-c, and Supplementary Tables 1-2).

For the larger monomeric BRAF complex containing MEK, the active sites of MEK and the BRAF KD are in a canonical face-to-face orientation, with the C-lobes of both kinases making extensive contacts and the activation segments running in an antiparallel manner (Fig. 2a and Supplementary Fig. 5d). As expected, MEK was in the inactive conformation with its $\alpha \mathrm{C}$-helix in 
the "out" position and density for the MEK inhibitor CH5126766 visible within the allosteric binding site (Supplementary Fig. 5d). In addition, residues of the $\alpha$ A-helix of MEK, which acts in a negative regulatory manner to stabilize the inactive conformation ${ }^{28}$, were resolved and lay against the base of the MEK $\alpha$ C-helix (Supplementary Fig. 5d).

Consistent with the previously published autoinhibited BRAF:14-3-32:MEK1 ${ }^{\text {S218A,S222A }}$ structure, the N-terminal segment of BRAF (amino acids 1-155) and the linker sequences between the CRD and the pS365 site (amino acids 281-359), and between the pS365 site and the kinase domain (amino acids 371-448) are not resolved in our monomeric structures, likely reflecting the flexibility of these regions. In contrast, the RBD is well-defined in our autoinhibited BRAF:14-3$3_{2}$ :MEK and BRAF:14-3-3 ${ }_{2}$ structures (Fig. 2a-b and Supplementary Fig. 4), revealing the position and orientation of this critical domain.

\section{RBD orientation and contacts in the autoinhibited monomeric BRAF complexes}

A distinct feature of the BRAF structures reported here is the resolution of the RBD in the context of the full-length, autoinhibited BRAF monomer. RBDs are known to have a conserved ubiquitinlike structure ${ }^{29}$ containing five beta strands and two to three alpha helices. As shown in Fig. 2a, the BRAF RBD in both monomeric structures sits on top of the 14-3-3 protomer bound to the Cterminal pS729 site and adjacent to the C-lobe of the KD, with N163 of the RBD being within hydrogen bond distance to S679 of the KD (Supplementary Fig. 6a). No RBD contact is observed with the CRD or with MEK in the MEK-bound structure. Notably, an extensive contact surface of $\sim 435 \AA^{2}$ is observed between the RBD and the 14-3-3 protomer, with the electrostatic charge distributions of the interface surfaces having considerable complementarity (Fig. 3a). The $\alpha 1$ helix of the RBD is oriented along the interface and interacts with the $\alpha 8$-helix, $\alpha 9$-helix, and loop 8 of 14-3-3 (Fig. 3b and Supplementary Fig. 6b). The 14-3-3 residues involved in this interface are conserved in all human 14-3-3 family members, indicating that a similar interface would be predicted regardless of the isoform composition of the 14-3-3 dimer. In contrast, two RBD 
residues at the center of the RBD:14-3-3 interface, M186 and M187, are not conserved, being replaced by a lysine and valine in CRAF and ARAF (Fig. 3b).

Because the M186 and M187 residues have the potential to make numerous contacts with 14-3-3 at the interface and given that contacts between 14-3-3 and the CRD are known to play a key role in maintaining the autoinhibited state, we next took a mutational approach to determine whether these RBD residues also contribute to RAF autoinhibition. M186 and M187 were mutated either to lysine and valine (as in CRAF and ARAF), to smaller alanine residues, to bulkier but still hydrophobic tryptophanes, or to negatively charged glutamic acid residues, following which the effect of these mutations on RAFautoinhibition was assessed using a proximity-based NanoBRET assay (Fig. 3c). In this assay, BRAF is split into two segments, with the regulatory domain (1 -435) tagged with an acceptor fluorophore (BRAFREG_Halo) and the kinase domain (436-766) tagged with an energy donor (NanoLuc-BRAFKD). When the two segments interact to form an autoinhibited complex that is stabilized by 14-3-3 dimer binding, the donor and acceptor are brought within range for energy transfer to occur $(<100 \AA)$, resulting in the generation of a BRET signal. As a control, we found that when the CRD of BRAFREG contained the RASopathyassociated T241P mutation, which reduces the autoinhibitory effect of the CRD, the BRET signal was decreased approximately 50\% (Fig. 3c). Analysis of the RBD mutants revealed that the M186K/M187V mutant generated a BRET signal that was equivalent to WT-BRAFREG. In contrast, a reproducible $10 \%$ decrease in signal was observed for the M186W/M187W mutant, whereas a $17 \%$ increase in signal was observed for the M186A/M187A mutant. Surprisingly, the glutamic acid substitutions did not disrupt BRAF autoinhibitory interactions but rather result in a reproducible 5-10\% increase in the BRET signal, which may reflect potential interactions with R222 in the 14-3-3 $\alpha 9$-helix. Both M186A/M187A-BRAFREG and M186E/M187E-BRAFREG were also found to be more effective at suppressing MEK activation mediated by the isolated BRAFKD protein than was WT-BRAF $^{\text {REG }}$ (Fig. 3d), indicating an enhanced ability to form a stable, autoinhibited BRAF complex. In addition, when incorporated into full-length BRAF, the M186W/M187W mutant exhibited an increased biological activity in focus forming assays (Fig. 
3e). Moreover, the activity of the RBD M186W/M187W and CRD T241P mutants in the focus forming assay correlated with the ability of these mutations to disrupt RAF autoinhibition in the BRET assay. Thus, while the CRD plays the predominant role in autoinhibition, these findings indicate that M186/M187 and the RBD also contribute to the maintenance of the autoinhibited state.

\section{Effects of RAS binding on the BRAF RBD:14-3-3 interface}

Under normal signaling conditions, the RAF activation process begins when an autoinhibited RAF monomer interacts with GTP-bound RAS ${ }^{9-11}$. Previously determined crystal structures of RASRBD complexes have shown that the $\beta 2$-strand and the $\alpha 1$-helix of the RBD interacts with the $\beta 2$ strand and the switch I region of RAS ${ }^{30,31}$ and that RAS-RBD binding involves ionic and hydrogen bonds as well as other Van der Walls interactions ${ }^{32}$. In our autoinhibited BRAF complexes, the RAS binding surface of the RBD faces away from the CRD and is oriented towards a space that we term the "RAS pocket" (Fig. 4a). This pocket is flanked by 14-3-3, the C-lobe of the KD, and MEK in the BRAF:14-3-32:MEK structure (Fig. 4a-b). Critical basic residues of the RBD, R158, R166, K183 and R188, are exposed and face towards the RAS pocket, ready to form ionic bonds with acidic residues E31, D33, E37 and D38 in RAS switch I (Fig. 4b).

To demonstrate that the RBD in our autoinhibited BRAF monomeric complexes is accessible for RAS binding, fluorescence polarization assays were conducted in which the purified BRAF:14-3-32:MEK and BRAF:14-3-32 complexes were titrated onto GFP-KRAS that had been pre-loaded with a GTP analog. As shown in Fig. 4c, both complexes exhibited a high degree of binding and had affinities in the nanomolar range. RAS-RAF binding could also be demonstrated in $\mathrm{KRAS}^{\mathrm{G} 12 \mathrm{~V}}$ pull-down assays using the purified BRAF:14-3-32:MEK and BRAF:14-3-32 complexes (Fig. 4d). As expected, mutation of any of the RBD basic residues that form ionic bonds with KRAS (R158A, R166A, K183, and R188L) significantly disrupted KRAS ${ }^{\mathrm{G} 12 \mathrm{~V}}$ binding in the pull-down assays (Fig. 4e), confirming that RBD contact is essential for the RAS-BRAF interaction. 
Although it has been known that binding to RAS relieves RAF autoinhibition and allows RAF to dimerize, the precise molecular details for how this is achieved has been unclear. Based on the known KRAS:RBD interface ${ }^{32}$, we superimposed KRAS onto our autoinhibited BRAF structures such that all of the ionic interactions needed for high affinity binding could form. Strikingly, in this position, a steric clash would occur between residues in the KRAS $\alpha 1$-helix and switch I region and the $\alpha 8$ - and $\alpha 9$ - helices of 14-3-3 that lie beneath the RBD and form the RBD:14-3-3 interface (Fig. 4f). Specifically, RAS residues I21, Q22 ( $\alpha 1$-helix), Q25-E31 (SI region), K42 and V45 ( $\beta 2$ sheet) would clash with 14-3-3 $\alpha 8$-helix residues C189, A192-E198, I200, A201 and $\alpha 9$-helix residues R222, L225, T226 and S230 (Supplementary Fig. 6c). Moreover, this region of 14-3-3 has a notable negative charge and, while complementary with the positively charged RBD interface, would cause electrostatic repulsion with negatively charged residues in KRAS switch I (Fig. 4f). In particular, KRAS switch I residues D30 and E31 would be brought in close proximity to D197 and E198 of 14-3-3. Thus, we propose the following model in which RAS-BRAF binding is a dynamic process and begins with the recruitment of the autoinhibited BRAF monomer to the membrane through interactions between RAS and the exposed basic residues of the RBD. Formation of the ionic bonds with RAS would generate steric clashes and electrostatic repulsion at the RBD:14-3-3 interface that would initiate a rearrangement in 14-3-3 dimer binding, resulting in the exposure of additional RBD residues involved in full RAS-RBD contact. We predict that the full spectrum of RAS-RBD interactions would dislodge the RBD from the autoinhibited complex as well as the CRD, due to the short linker between these two domains, thereby enabling the CRD to rotate and make contact with RAS and the plasma membrane. This structural reoganization, in turn, would promote the release of 14-3-3 from the pS365 site and expose the BRAF dimer interface for dimer formation and catalytic activation.

In support of this model, our results below indicate that the RBD M186/M187 residues at the center of RBD:14-3-3 interface serve a dual role by mediating contact with 14-3-3 in the autoinhibited state and by contributing to RAS binding interactions upon RAS activation. These methionines are a lysine and valine in CRAF, and structural studies indicate that K87 and V88 of 
CRAF contributes to full RAS:RBD contact by forming extensive non-electrostatic interactions with KRAS residues I24, Q25, and Y4032. Although M186 and M187 in BRAF are partially or fully occluded by 14-3-3 in the autoinhibited state, the steric clash caused by initial RAS:RBD contact would directly impact 14-3-3 residues that interact with these methionines, perhaps resulting in their exposure for contact with RAS. Therefore, to address whether these BRAF residues contribute to the RAS-RBD interaction, the M186/M187 mutants were assessed for binding to KRAS $\mathrm{K}^{\mathrm{G}} \mathrm{V}$ in co-immunoprecipitation experiments and in a BRET assay that monitors RAS-RAF binding in live cells. In particular, the BRET assay allows for RAS-RAF binding levels $\left(\mathrm{BRET}_{\max }\right)$ and for the affinity of the interaction $\left(\mathrm{BRET}_{50}\right)$ to be quantified when saturation curves are performed ${ }^{33}$. From these assays, we found that mutation of M186/M187 to alanine or glutamic acid residues reduced binding to KRAS ${ }^{\mathrm{G} 12 \mathrm{~V}}$ (Fig. 5a-b), with the glutamic acid substitutions having the most pronounced effect. In contrast, when M186/M187 were mutated to bulkier tryptophan residues (M186W/M187W) or changed to lysine and valine (M186K/M187V), as in CRAF, increased levels of binding to KRASG12V were observed in both the BRET and coimmunoprecipitation assays (Fig. 5a-b).

To test whether the modulation in binding reflects direct RAS-RBD contact, RBD pulldown experiments were performed using isolated WT or mutant BRAF RBD domains. As was observed for the full-length BRAF proteins, the M186W/M197W and the M186K/M187V mutants exhibited increased binding to $\operatorname{KRAS}^{\mathrm{G} 12 \mathrm{~V}}$ (Fig. 5c), whereas lower binding levels were observed for the M186A/M187A mutant, and little to no binding was detected for the M186E/M187E mutant, indicating that the mutations were directly influencing interactions between the BRAF RBD and KRASG12V. Modeling of the amino acid substitutions (Fig. 5d-e) into the K87/V88 positions of a CRAF RBD:RAS structure ${ }^{32}$ further supports these findings in that while the KV/MM substitutions would change some of the backbone and side chain interactions, non-eletrostatic interactions would still be predicted. With the KV/WW mutations, the bulkier tryptophan residues would have the potential to form additional interactions, whereas the alanine substitutions would result in the loss of all side chain contacts. For the KV/EE 
mutations, the negatively charged glutamic acid residues would cause electrostatic repulsion with RAS residues E31 and D33 and be inhibitory to binding. Thus, taken together our mutational analysis indicates that these central residues in the BRAF RBD:14-3-3 interface serve dual functions in first contributing to the autoinhibited state through interactions with 14-3-3 and then in contributing to the full spectrum of RAS-RBD interactions that facilitates the conformation changes needed to disrupt the autoinhibited state.

\section{Discussion}

It is well established that under most signaling conditions binding to activated RAS is required for RAF dimerization and activation; however, the structural details for how RAS binding allows autoinhibited RAF monomers to assume an active dimer conformation has been unclear. In this study, we successfully obtained cryo-EM structures of autoinhibited, monomeric BRAF complexes (BRAF:14-3-32:MEK and BRAF:14-3-3 2 at 3.7 and 4.1 A resolution respectively) and a RAF inhibitor-bound dimeric complex (BRAF $2: 14-3-3_{2}$ at $3.9 \AA$ resolution). These complexes were isolated from a mammalian cell expression system such that components of the complex would be subject to normal cellular regulation and more closely represent physiological signaling complexes. The autoinhibited BRAF structures show that prior to signaling events, BRAF and its substrate MEK can exist as a preassembled BRAF:14-3-32:MEK complex; however, the interaction with MEK is not required for BRAF to maintain the autoinhibited conformation. This finding is consistent with a previous study which found that BRAF was still present in the flow through of human 293 cell lysates where MEK had been immunodepleted, suggesting a dymanic equilibrium between MEK-bound and MEK-free BRAF complexes ${ }^{34}$. Notably, both of our autoinhibited BRAF structures had clearly resolved RBDs, revealing the position and orientation of this critical domain and providing valuable insight regarding how RAS binding facilitates the monomer to dimer transition.

The position and orientation of the RBD in the context of the autoinhibited BRAF complexes indicates that the RBD is accessible for RAS binding, with key basic residues involved 
in RAS contact exposed. However, it should be noted that the large BRAF specific, N-terminal segment that precedes the BRAF RBD was not resolved in our structures. Thus, it is possible that this region, may represent an additional level of regulation in terms of the RAS-BRAF interaction, as has been suggested in recent live cell studies analyzing the binding preferences of the RAS and RAF family members ${ }^{33}$. Nonetheless, both the purified BRAF:14-3-32:MEK and BRAF:14-3-32 complexes were fully competent to bind GTP-bound KRAS in fluorescence polarization assays and displayed high affinity binding. Our autoinhibited structures also revealed a significant contact interface between the RBD and the 14-3-3 protomer bound to the C-terminal pS729 site. The interface surfaces had charge complementarity, with the RBD surface containing several positively charged residues and the 14-3-3 surface having a predominant negative charge. The 143-3 residues that comprise the interaction surface are conserved in all 14-3-3 family members, indicating that the surface would be the same irrespective of which isoform was bound.

Strikingly, our results indicate that two RBD residues at the center of RBD:14-3-3 interface, M186/M187, also contribute to RAS binding and may play a key role in facilitating the conformational changes in 14-3-3 binding that are needed for dimer formation. Recent crystal structures of CRAF RBD and CRAF RBD-CRD in complex with KRAS indicate that CRAF residues (CRAF K87/V88) at analogous positions to the BRAF methionines make van der Waals contacts with residues I24/Q25/Y40 in $\mathrm{RAS}^{32}$. Although no structure of RAS in complex with the BRAF RBD currently exists, our mutational analysis of the BRAF RBD M186/M187 residues suggests that alterations in these residues can modulate KRAS binding. Thus, integrating our autoinhibited BRAF structures into what is known regarding the RAS-RBD interaction strongly supports a model whereby RAS binding is a highly dynamic process (Fig. 6). The initial contact with activated RAS is likely made by exposed basic residues in the BRAF RBD (R158, R166, K183, R188). As RAS is engaged to form the high affinity ionic bonds, a steric clash and electrostatic repulsion between RAS and 14-3-3 would occur at the RBD:14-3-3 interface, leading to the exposure of additional RBD residues involved in the full spectrum of RAS-RBD interactions. We predict that together these events will dislodge the RBD and in turn the CRD from the 
autoinhibited structure, thus putting further strain on the bound 14-3-3 dimer that ultimately results in its release from the pS365 site. The freed CRD could then contact RAS and the plasma membrane to further stabilize RAS-RAF complexes and aid in orienting the KD for dimer formation. It should be noted that crystal structures of RAS bound to a truncated CRAF protein containing only the RBD-CRD have recently been reported (HRAS:CRAF_RBD-CRD ${ }^{35}$ and KRAS:CRAF_RBD-CRD complexes ${ }^{32}$ ) and demonstrate significant contact of the CRAF CRD with RAS. Based on the position and orientation of the RBD and CRD in our autoinhibited BRAF structures, it appears that if the BRAF CRD makes similar contacts with activated RAS, the CRD would need to rotate as it is dislodged from autoinhibited complex in ord er to assume the final RAS binding state. More specifically, when the bound RBD-CRD structure is overlayed onto our autoinhibited structure, with the alignment based on the CRD position, the RBD of the CRAF_RBD-CRD structure would lie outside the cryo-EM density map (Supplementary Fig. 4g). Thus, the RAS:CRAF_RBD-CRD structure may be more representative of the final RAS-BRAF binding conformation rather than the initial contact between RAS and the autoinhibited BRAF complexes.

Not surprising, given the critical role of the membrane environment in forming the active dimeric RAF complex, the entire regulatory region of BRAF was unstructured in our dimeric $\mathrm{BRAF}_{2}: 14-3-3_{2}$ complex. As RAF inhibitor treatment was used to facilitate the isolation of dimer complexes, SB590885 was detected in the ATP-binding pocket of both KD protomers. Each KD protomer also interacted with a protomer of a 14-3-3 dimer, which was bound to the C-terminal pS729 site. The orientation between the KD and 14-3-3 dimers was asymmetric and similar to that observed in the other structures of dimerized BRAF KDs bound to 14-3-318-20. This asymmetry was initially proposed to result from the insertion of a C-tail segment from one BRAF KD protomer into the active site of the other ${ }^{18}$; however, the fact that a similar orientation has been observed when the active sites are fully occupied by RAF inhibitors or when the KDs lack the Cterminal segments, has argued against this model ${ }^{19,20}$. 
Finally, comparison of the KD conformation in inhibitor-bound BRAF dimer complexes with that of our autoinhibited, monomeric BRAF structures is in agreement with previous observations that the $\mathrm{N}$ - and $\mathrm{C}$-lobes of the inhibitor-bound dimerized complexes are in a more open configuration ${ }^{17,20}$. Interestingly, in our monomeric BRAF structures, this compact kinase domain configuration, which is known to put the dimer interface in an unfavorable position to form the $\mathrm{N}$ - to $\mathrm{C}$-lobe antiparallel interactions needed to form dimers, was observed in the absence of ATP binding, differing from the previously reported structures exhibiting this conformation ${ }^{17,20}$. Nonetheless, ourfindings are in agreement with the model that identifying compounds or strategies to stabilize this compact configuration may have therapeutic potential in blocking aberrant RAF dimerization.

In summary, our studies contribute to the understanding of the BRAF signaling process by providing biochemical and structural information regarding different BRAF states. Further investigation is still needed to determine the mechanistic details for the transition between states and how these transitions are altered by events such as phosphorylation, dephosphorylation, changes in the signaling environment, and interactions with other binding partners. Moreover, it will be important to determine if other RAF family members have similar monomer and dimer architectures and whether heterodimer complexes may differ. This knowledge will provide a more mechanistic understanding of the signaling process and may aid in the development of more effective treatment strategies for RAS- and/or RAF-driven disease states.

\section{Methods}

\section{Reagent or Resource}

Resources used in this study are listed in Supplementary Table 3.

\section{Cell Lines and Culture Conditions}

293FT, 293T, HeLa, NIH-3T3, and Phoenix-Eco cells were cultured in DMEM supplemented with $10 \%$ fetal bovine serum (FBS), 2 mM L-glutamine, and 1\% penicillin/streptomycin. All cells were cultured at $37^{\circ} \mathrm{C}$ with $5 \% \mathrm{CO}_{2}$. 


\section{Methods Details}

\section{Generation of Recombinant Lentivirus and Stable 293FT Cells Expressing Halo-BRAF ${ }^{\mathrm{WT}}$}

Recombinant lentivirus particles were generated by co-transfecting psPAX2 and pMD2 with the pLenti Halo-BRAFWT construct into 293T cells using the X-tremeGENE9 transfection reagent. 72 hours post-transfection, the virus-containing supernatant was collected, centrifuged twice at $935 \mathrm{x}$ $\mathrm{g}$ for 10 minutes to remove any cellular debris, and then stored at $-80^{\circ} \mathrm{C}$. $293 \mathrm{FT}$ cells were infected with viral supernatants containing $8 \mu \mathrm{g} / \mathrm{mL}$ polybrene for 24 hours, following which growth media containing $2 \mu \mathrm{g} / \mathrm{mL}$ puromycin (selection media) was added. The selection media was changed every 3 days until resistant cells were obtained.

\section{Affinity Purification of BRAF Complexes and Size Exclusion Chromatography}

Halo-BRAFWT 293FT cells were plated into forty-five $10 \mathrm{~cm}$ tissue culture dishes at a concentration of $2 \times 10^{6}$ cells/dish and allowed to grow to confluence ( $\sim 3$ days after plating). For BRAF monomer samples, media was left unchanged until collection to allow for serum-depletion. For BRAF dimer samples, media was replaced the day prior to collection with fresh growth media containing $2 \mu \mathrm{M}$ SB590885. On the day of collection, media was removed by suction from each plate and the cells were washed twice with $5 \mathrm{~mL}$ cold PBS. Cells were then lysed in Triton lysis buffer (1\% Triton X-100, $137 \mathrm{mM} \mathrm{NaCl}, 20 \mathrm{mM}$ Tris $\mathrm{pH} 8.0,0.15 \mathrm{U} / \mathrm{mL}$ aprotinin, $1 \mathrm{mM}$ phenylmethylsulfonyl fluoride, $20 \mu \mathrm{M}$ leupeptin; $0.5 \mathrm{mM}$ sodium vanadate; $1 \mathrm{~mL}$ per $10 \mathrm{~cm}$ dish) at $4{ }^{\circ} \mathrm{C}$ for 15 minutes on a rocking platform. Lysates were collected and clarified of debris by centrifugation at $16,100 \times \mathrm{g}$ for 10 minutes at $4{ }^{\circ} \mathrm{C}$.

To isolate the BRAF complexes, $5 \mathrm{~mL}$ of Halolink resin (Promega) was washed twice with Triton X-100 lysis buffer prior to the addition of lysate. The samples were then incubated for 2 hours at $4^{\circ} \mathrm{C}$ on a rocking platform. Beads containing the bound BRAF complexes were washed twice with Triton X-100 lysis buffer and three times with elution buffer (137 mM NaCl and 20 $\mathrm{mM}$ Tris $\mathrm{pH}$ 8.0). The bead-bound complexes were then resuspended in $2.5 \mathrm{~mL}$ elution buffer containing $50 \mu \mathrm{L}$ Halo-TEV (Promega) and incubated for 2 hours at $4^{\circ} \mathrm{C}$ on a rocking platform. 
The beads were then pelleted, and the supernatant containing the eluted BRAF complexes was applied to a Superdex 6 Increase 10/300 GL column (Cytiva) pre-equilibrated with a buffer containing $20 \mathrm{mM}$ Tris $\mathrm{pH} 8.0$ and $137 \mathrm{mM} \mathrm{NaCl}$. Proteins from the peak fractions corresponding to BRAF complexes were collected and analyzed by SDS-PAGE and silver staining.

\section{Cryo-EM Grid Preparation, Data Acquisition, and Processing}

After gel filtration, the BRAF:14:3-32:MEK, BRAF:14-3-3 ${ }_{2}$ and $\mathrm{BRAF}_{2}: 14-3-3_{2}$ complexes (in 20 $\mathrm{mM}$ Tris $\mathrm{pH} 8.0$ and $137 \mathrm{mM} \mathrm{NaCl}$ ) were concentrated to $0.18-0.20 \mathrm{mg} / \mathrm{mL}$ by centrifugation in a $30 \mathrm{~K}$ pore size Pall's Microsep ${ }^{\mathrm{TM}}$ advance centrifugal device. The samples were supplemented with $\mathrm{NaCl}$ and DTT to a final concentration of $200 \mathrm{mM}$ and $10 \mathrm{mM}$, respectively, before freezing. Quantifoil Au R 1.2/1.3 holey carbon girds were glow-discharged for 45 seconds at $20 \mathrm{~mA}$ in an alcohol environment. A volume of $1.5 \mu \mathrm{L}$ of protein solution was applied to each side of the grids, and the samples were vitrified in a FEI Vitrobot Mark IV, using a force of 10 au and a blotting time of 1.5 to 2.0 seconds at $4{ }^{\circ} \mathrm{C}$ with humidity $>85 \%$. The samples were then frozen by plungefreezing the grids into liquid ethane cooled to approximately $-180^{\circ} \mathrm{C}$ by liquid nitrogen. $50 \mathrm{frames}$ per movie were collected from the frozen hydrated samples at a nominal magnification of 130,000x for the BRAF:14:3-32:MEK sample (corresponding to $1.058 \AA$ per physical pixel) using a Titan Kiros electron microscope (FEI) at $300 \mathrm{kV}$ with a $\mathrm{K} 2$ summit direct detection camera (Gatan) in super-resolution mode. The slit width of the energy filter was set to $20 \mathrm{eV}$. Then, using SerialEM ${ }^{36}$ data collection software, the micrographs were dose-fractionated into 50 frames with a total exposure time of 8 seconds and a total electron exposure of 57 electrons per $\AA^{2}$, with defocus values ranging from -0.8 to $-2.5 \mu \mathrm{M}$.

All cryo-EM data analysis was done using RELION 3.1 ${ }^{37}$. For the BRAF:14:3-32:MEK complex, a total of 3,976 micrographs were collected. The raw movies were aligned and gain corrected to compensate for sample movement and drift by MotionCor $2^{38}$ with 5 by 5 patches and a B-factor of 150. The Getf program ${ }^{39}$ was used to estimate the contrast transfer function (CTF) parameters of each motion-corrected image. The micrographs were screened to remove low- 
quality images and those with unqualified CTF power spectra. An initial set of particles were manually picked and used to generate 2D class templates for automatic particle picking, using a box size of 180 pixels. Particle selection was inspected to remove any contaminants and to add missed particles. The resulting particles were subjected to three rounds of $2 \mathrm{D}$ classification to identify and discard false positives or other apparent contaminants. Following 2D classification, particles were further selected, re-centered, and re-extracted for 3D classification. An initial model was generated and, based on structural integrity and map quality of complex, the best 3D class was then used for 3D refinement. After 3D auto-refinement, Bayesian polishing and per-particle CTF refinements were applied, until the 3D refinement converged. The final map was sharpened using a B-factor of $-100.5 \AA^{2}$, yielding a density map at a resolution of $3.7 \AA$, based on the gold standard FSC 0.143 criteria. Masked-based local refinement was also performed on the BRAF KD:MEK portion of the structure, which generated a density map with the same resolution and quality, and on the BRAF KD portion of the structure, which resulted in no further improvement of the map.

The monomeric BRAF:14-3-3 3 and dimeric $\mathrm{BRAF}_{2}: 14-3-3_{2}$ datasets were collected in a similar manner, with 50 frames per movie collected from the frozen hydrated samples at a nominal magnification of $105,000 \mathrm{x}$ (corresponding to $1.348 \AA$ per physical pixel). The micrographs were dose-fractionated into 50 frames every 0.2 seconds with a dose rate of $5.5 \mathrm{e}-/ \AA^{2} / \mathrm{s}$, total exposure time of 10 seconds and an accumulated dose of 55 electrons per $\AA^{2}$. The datasets were further processed in the same manner as described for the BRAF:14-3-32:MEK dataset Supplementary Table 4.

Models for the autoinhibited BRAF complexes were built by rigid-body fitting of the individual BRAF domains using PDB IDs $5 \mathrm{~J} 17$ for the RBD ${ }^{40}$, 1FAR for the CRD ${ }^{41}, 4 \mathrm{MNE}$ for the $\mathrm{KD}^{34}$, and $3 \mathrm{WIG}^{42}$ for MEK1 protomer, when present. Similarly, the active BRAF dimer complex was built by rigid-body fitting using PDB ID $2 F B 8^{43}$ for the BRAFKD. For all structures, 14-3-3 was built by rigid-body fitting using 14-3-3 $\zeta$ PDB ID 4FJ344. Each subunit of 14-3-3 dimer was fit individually, and the BRAF pS365 and pS729 sites were built manually as were any additional residues needed. Fitting of the models into their respective maps was initially done 
using UCSF Chimera ${ }^{45}$. Manual adjustment of the model was performed in $\operatorname{Coot}^{46}$, followed by iterative rounds of real space refinement in Phenix ${ }^{47}$ and manual fitting in Coot. Model validation was done using statistics from Ramachandran plots and MolProbity scores in Phenix and Coot. Statistics for the final refinements are shown in Supplementary Table 4. Figures were generated by UCSF Chimera. Structure deviations and electrostatic potential of surfaces were calculated using MatchMaker and Columbic Surface plugins, respectively, in UCSF Chimera.

Initial diagnostic data sets for the three BRAF complexes were collected on a Talos Arctica microscope (FEI) at $200 \mathrm{Kv}$ with a Gatan K3 summit direct detection camera at either the NCIFrederick cryo-EM Facility or at the John M. Cowley Center for High Resolution Electron Microscopy, Arizona State University. Data sets for generating the structures with the final reported resolutions were collected on a Titan Krios microscope at $300 \mathrm{kV}$ with a Gatan K2 summit direct detection camera in super-resolution mode.

\section{Fluorescence Polarization Assay}

Fluorescence polarization assay was used to measure the binding of BRAF:14-3-32:MEK and BRAF:14-3-3 2 complexes to KRAS. GppNHp-loaded GFP-KRAS at concentration of $0.73 \mathrm{nM}$ was mixed with 0.0057-2.4 $\mu \mathrm{M}$ of BRAF:14-3-32:MEK and BRAF:14-3-3 2 complexes in buffer containing $20 \mathrm{mM}$ Tris $\mathrm{pH} 8.0$ and $137 \mathrm{mM} \mathrm{NaCl}$. Fluorescence polarization of the samples was measured in black flat-bottom assay plates (Corning) using a CLARIOstar micro-plate reader (BMG LABTECH) with $482 \mathrm{~nm}$ excitation and $540 \mathrm{~nm}$ emission. The data was analyzed and fitted to the anisotropy single association hyperbolic equation using Prism 8 software.

\section{NanoBRET RAF Autoinhibition Assay}

293FT cells were seeded into 6-well tissue culture plates at a concentration of 4 x $10^{5}$ cells/well. 16 hours after plating, cells were co-transfected using $5 \mathrm{ng}$ of the indicated NanoLuc-RAFCAT construct and $20 \mathrm{ng}$ of the indicated BRAFREG-Halo construct (per well). Twenty-four hours later, cells were collected and resuspended in serum-free/phenol free Opti-MEM (Gibco). HaloTag 618 ligand was added to the cell suspension $(1 \mu \mathrm{L} / \mathrm{mL})$, and cells were seeded in quadruplicate into 
wells of a 384-well plate (BioTek) at a concentration of $8 \times 10^{3}$ cells per well, with the remaining cells plated into a fresh 6 -well tissue culture plate. Cells were incubated at $37^{\circ} \mathrm{C}$ with $5 \% \mathrm{CO}_{2}$ for 24 hours, following which NanoGlo substrate was added to each well of cells $(10 \mu \mathrm{L} / \mathrm{mL})$, and the donor (460nm) and acceptor (618nm) emissions were measured using a Perkins Elmer Envision plate reader (\#2104-0010A containing a 460nm/50nm emission filter and a 610nm LPfilter). Cells seeded into the 6-well plates were lysed and examined by immunoblot analysis using antibodies recognizing the Halo-tag (for RAFREG detection) and NanoLuc-tag (for RAFCAT detection) to ensure equal expression levels.

\section{Transfection, Cell Lysis, and Co-immunoprecipitation Assays}

HeLa or 293FT cells were plated into $10 \mathrm{~cm}$ tissue culture dishes at a concentration of $0.5 \mathrm{x}$ 106/dish, 18-24 hours prior to transfection. Cells were then transfected using the XtremeGENE9 transfection reagent, per the manufacturer's instructions, at a 2:1 ratio of XtremeGENE9 to DNA. 30 hours after transfection, cells were serum-starved for 18 hours, prior to lysis. For cell lysis, cells were washed twice with ice cold PBS and lysed in 1\% NP-40 buffer (20 mM Tris pH 8.0, $137 \mathrm{mM} \mathrm{NaCl}, 10 \%$ glycerol, $1 \% \mathrm{NP}-40$ alternative, $0.15 \mathrm{U} / \mathrm{mL}$ aprotinin, $1 \mathrm{mM}$ phenylmethylsulfonyl fluoride, $0.5 \mathrm{mM}$ sodium vanadate, and $20 \mu \mathrm{M}$ leupeptin) at $4^{\circ} \mathrm{C}$ for 15 minutes on a rocking platform. Lysates were clarified by centrifugation at $16,100 \mathrm{x} g$ for 10 minutes at $4^{\circ} \mathrm{C}$, following which the protein content was determined by Bradford assays. Lysates containing equivalent amounts of protein were incubated with the appropriate antibody and protein $\mathrm{G}$ sepharose beads for 2 hours at $4^{\circ} \mathrm{C}$ on a rocking platform. Complexes were washed extensively with $1 \%$ NP-40 buffer and then examined by immunoblot analysis, together with aliquots of equalized total cell lysate.

\section{Focus Forming Assay}

Recombinant retroviruses expressing the RAF proteins of interest were generated by transfecting $6 \mu \mathrm{g}$ of the indicated pBabe DNA construct into a $100 \mathrm{~mm}$ tissue culture dish of Phoenix-Eco cells using the XtremeGENE9 protocol described above. Viral supernatants were collected 48 hours 
post-transfection, centrifuged twice at $526 \mathrm{x}$ g for 10 minutes, and either stored at $-80^{\circ} \mathrm{C}$ or used directly. NIH-3T3 cells were plated into $60 \mathrm{~mm}$ tissue culture dishes at a concentration of $2 \times 10^{5}$ cells/dish. After 16 hours, cells were infected with the indicated recombinant retrovirus in media containing 4\% FBS and $8 \mu \mathrm{g} / \mathrm{mL}$ polybrene. 24 hours later, cells were trypsinized and plated into two $100 \mathrm{~mm}$ dishes, one of which contained $5 \mu \mathrm{g} / \mathrm{mL}$ puromycin. After $2-4$ weeks of culture, cells were fixed with $3.7 \%$ formaldehyde and stained with $1 \%$ methylene blue.

\section{Pull down Assays Using Halo-RAS or Halo-RBD Beads}

For bead preparation, 293FT cells transiently expressing the indicated Halo-KRAS ${ }^{\mathrm{G} 12 \mathrm{~V}}$ or HaloRBD constructs were lysed in RIPA lysis buffer (20 mM Tris pH 8.0, 137 mM NaCl, 10\% glycerol, $1 \%$ NP-40 alternative, $1 \% \mathrm{SDS}, 0.1 \%$ sodium deoxycholate, $0.15 \mathrm{U} / \mathrm{mL}$ ap rotinin, $1 \mathrm{mM}$ phenylmethylsulfonyl fluoride, $0.5 \mathrm{mM}$ sodium vanadate, and $20 \mathrm{mM}$ leupeptin; $1 \mathrm{~mL}$ per $10 \mathrm{~cm}$ dish) at $4^{\circ} \mathrm{C}$ for 15 minutes on a rocking platform. Lysates were clarified by centrifugation at $16,100 \mathrm{x}$ g for $10 \mathrm{~min}$ at $4^{\circ} \mathrm{C}$. Halolink resin (Promega) was washed twice with RIPA buffer and then added to the lysate ( $30 \mu \mathrm{L}$ of bead resin per $\mathrm{mL}$ of lysate). Samples were incubated at $4{ }^{\circ} \mathrm{C}$ for 2 hours on a rocking platform. Beads containing the Halo-tagged proteins were then washed once with RIPA buffer and twice with Triton lysis buffer (1\% Triton, $137 \mathrm{mM} \mathrm{NaCl}, 20 \mathrm{mM}$ Tris $\mathrm{pH}$ 8.0), prior to resuspension in Triton $\mathrm{X}-100$ lysis buffer.

For pull-down assays using cell ly sates, 293FT cells expressing the desired RAS or BRAF proteins were lysed in Triton X-100 lysis buffer, following which the protein content of the lysates was determined by Bradford assays. Lysates containing equivalent amounts of protein were incubated with the Halo-RAS or Halo-RBD beads ( $30 \mu \mathrm{L}$ beads per $1 \mathrm{~mL}$ lysate) at $4^{\circ} \mathrm{C}$ for 1.5 hours on a rocking platform. The beads containing the pull-down complexes were washed once with Triton lysis buffer and twice with elution buffer (137 $\mathrm{mM} \mathrm{NaCl}$ and $20 \mathrm{~m} \mathrm{M}$ Tris $\mathrm{pH}$ 8). The bead pellet was resuspended in $150 \mu \mathrm{L}$ elution buffer containing $2 \mu \mathrm{L}$ Halo-TEV (Promega) and incubated at $4^{\circ} \mathrm{C}$ for 30 minutes. Beads were then pelleted and the eluate collected for immunoblot analysis. For pull-down experiments using the purified BRAF:14-3-3 2 and BRAF:14-3-32:MEK 
complexes, $500 \mu \mathrm{L}$ of the gel filtration fractions containing these complexes was incubated with $30 \mu \mathrm{L}$ of washed $\mathrm{KRAS}^{\mathrm{G} 12 \mathrm{~V}}$ beads at $4^{\circ} \mathrm{C}$ for 1.5 hours on a rocking platform. Beads were then washed three times with elution buffer, and the eluate collected as described above.

\section{BRET RAS-RAF Interaction Assay}

293FT cells were seeded into 12 -well dishes at a concentration of $1 \times 10^{5}$ cells/well. 16 hours after plating, Venus-tagged and Rluc8-tagged constructs were co-transfected into cells using a calcium phosphate protocol. Duplicate 12-point saturation curve was generated in which the concentration of the energy donor construct (Rluc8) was held constant $(62.5 \mathrm{ng})$ as the concentration of the energy acceptor plasmid (Venus) increased (0-1.0 $\mu \mathrm{g})$. Cells were collected 48 hours after transfection, washed, and resuspended in PBS $(500 \mu \mathrm{L}) .30 \mu \mathrm{L}$ of the cell suspension was plated in duplicate into wells of a 384-well white-walled plate (PerkinElmer CulturPlate) and coelenterazine-h was added to a final concentration of $3.375 \mu \mathrm{M}$. The BRET and Rluc 8 emissions were measured simultaneously using a PHERAstar Plus plate reader (BMG Labtech), with BRET monitored at $535 \mathrm{~nm}$ (bandwidth $30 \mathrm{~nm}$ ) and Rluc8 measured at $475 \mathrm{~nm}$ (bandwidth $30 \mathrm{~nm}$ ). To monitor the increasing levels of acceptor expression, $90 \mu \mathrm{L}$ of the cell suspension was also plated in duplicate into wells of a 96-well black-walled plate (PerkinElmer OptiPlate), and Venus fluorescence was determined using an excitation wavelength of $485 \mathrm{~nm}$ (5 $\mathrm{nm}$ bandwidth) and the emission monitored at $530 \mathrm{~nm}$ (5 nm bandwidth) using a Tecan Infinite M1000 plate reader. The BRET ratio was determined by calculating the 535/475 ratio for a given sample (sample BRET) and was normalized to the BRET signal from cells expressing the donor construct alone to enable comparisons between experiments. The normalized mBRET signal was calculated as follows: (1000 X (sample BRET ratio/background BRET ratio)) - 1000. The acceptor/donor ratio (Venus emission/Rluc8 emission) for each data point was background corrected and equalized against a control where equal quantities of the Venus (acceptor) and Rluc8 (donor) constructs were transfected to allow for comparisons between experiments. Data was analyzed using GraphPad 
Prism. Non-linear regression was used to plot the best fit hyperbolic curve, and values for $\mathrm{BRET}_{\max }$ and BRET 50 were obtained from the calculated best fit curves.

\section{Data Availability}

Three-dimensional cryo-EM density maps have been deposited in the Electron Microscopy data Bank under accession numbers EMD-23813 (BRAF:14-3-32:MEK), EMD-23814 (BRAF:14-3$\left.3_{2}\right)$, and EMD-23815 (BRAF $\left.: 14-3-3_{2}\right)$. The coordinates of atomic models have been deposited in the Protein Data Bank under accession numbers PDB ID: 7MFD (BRAF:14-3-32:MEK) [https://www.rcsb.org/structure/unreleased/7MFD], $7 \mathrm{MFE}$

(BRAF:14-3-32)

[https://www.rcsb.org/structure/unreleased/7MFE], and 7MFF $\quad\left(\mathrm{BRAF}_{2}: 14-3-3_{2}\right)$ [https://www.rcsb.org/structure/unreleased/7MFF]. Source data are provided with this paper.

\section{Lead Contact and Materials Availability}

Further information and requests for resources and reagents should be directed to and will be fulfilled by the Lead Contacts, Deborah Morrison (ㅆmorrisod@mail.nih.gov) and Ping Zhang (ping.zhang@nih.gov). Plasmids and cell lines are available for use upon request to the Lead Contacts.

\section{References}

1 Cox, A. D. \& Der, C. J. Ras history: The saga continues. Small GTPases 1, 2-27, doi:10.4161/sgtp.1.1.12178(2010).

2 Lavoie, H. \& Therrien, M. Regulation of RAF protein kinases in ERK signalling. Nature reviews. Molecular cell biology 16, 281-298, doi:10.1038/nrm3979(2015).

3 Terrell, E. M. \& Morrison, D. K. Ras-Mediated Activation of the Raf Family Kinases. Cold Spring Harbor perspectives in medicine 9, doi:10.1101/cshperspect.a033746 (2019).

4 Nan, X. et al. Single-molecule superresolution imaging allows quantitative analysis of RAF multimer formation and signaling. Proceedings of the National Academy of Sciences of the United States of America 110, 18519-18524, doi:10.1073/pnas.1318188110 (2013).

5 Cutler, R. E. J., Stephens, R. M., Saracino, M. R. \& Morrison, D. K. Autoregulation of the Raf-1 serine/threonine kinase. Proc Natl Acad Sci USA 95, 9214-9219 (1998).

6 Guan, K.-L. et al. Negative regulation of the serine/threonine kinase B-Raf by Akt. J. Biol. Chem. 275, 27354-27359 (2000).

7 Tran, N. H., Wu, X. \& Frost, J. A. B-Raf and Raf-1 are regulated by distinct autoregulatory mechanisms. J Biol Chem 280, 16244-16253, doi:10.1074/jbc.M501185200 (2005). 
8 Freeman, A. K. \& Morrison, D. K. 14-3-3 Proteins: diverse function sin cell proliferation and cancer progression. Semin Cell Dev Biol 22, 681-687, doi:10.1016/j.semcdb.2011.08.009 (2011).

9 Van Aelst, L., Barr, M., Marcus, S., Polverino, A. \& Wigler, M. Complex formation between Ras and Raf and other protein kinases. Proc. Natl. Acad. Sci. USA 90, 6213-6217 (1993).

10 Vojtek, A. B., Hollenberg, S. M. \& Cooper, J. A. Mammalian Ras interacts directly with the serine/threonine kinase Raf. Cell 74, 205-214 (1993).

11 Zhang, X. F. et al. Normal and oncogenic $\mathrm{p} 21$ ras proteins bind to the amino-terminal regulatory domain of c-Raf-1. Nature 364,308-313, doi:10.1038/364308a0 (1993).

12 Marais, R., Light, Y., Paterson, H. F. \& Marshall, C. J. Ras recruits Raf-1 to the plasma membrane for activation by tyrosine phosphorylation. Embo j 14, 3136-3145 (1995).

13 Weber, C. K., Slupsky, J. R., Kalmes, H. A. \& Rapp, U. R. Active Ras induces heterodimerization of cRaf and BRaf. Cancer Res 61, 3595-3598 (2001).

14 Rushworth, L. K., Hindley, A. D., O'Neill, E. \& Kolch, W. Regulation and role of Raf-1/BRaf heterodimerization. Mol Cell Biol 26, 2262-2272, doi:10.1128/mcb.26.6.22622272.2006 (2006).

15 Freeman, A. K., Ritt, D. A. \& Morrison, D. K. Effects of Raf dimerization and its inhibition on normal and disease-associated Raf signaling. Mol Cell 49, 751-758, doi:10.1016/j.molcel.2012.12.018 (2013).

$16 \mathrm{Hu}$, J. et al. Allosteric activation of functionally asymmetric RAF kinase dimers. Cell 154, 1036-1046, doi:10.1016/j.cell.2013.07.046(2013).

17 Park, E. et al. Architecture of autoinhibited and active BRAF-MEK1-14-3-3 complexes. Nature, doi:10.1038/s41586-019-1660-y (2019).

18 Kondo, Y. et al. Cryo-EM structure of a dimeric B-Raf:14-3-3 complex reveals asy mmetry in the active sites of B-Raf kinases. Science 366, 109-115, doi:10.1126/science.aay0543 (2019).

19 Liau, N. P. D. et al. Dimerization Induced by C-Terminal 14-3-3 Binding Is Sufficient for BRAF Kinase Activation. Biochemistry 59,3982-3992, doi:10.1021/acs.biochem.0c00517 (2020).

20 Liau, N. P. D. et al. Negative regulation of RAF kinase activity by ATP is overcome by 14-3-3-induced dimerization. Nat Struct Mol Biol 27, 134-141, doi:10.1038/s41594-0190365-0 (2020).

21 Geiger, T., Wehner, A., Schaab, C., Cox, J. \& Mann, M. Comparative proteomic analysis of eleven common cell lines reveals ubiquitous but varying expression of most proteins. Mol Cell Proteomics 11, M111.014050, doi:10.1074/mcp.M111.014050 (2012).

22 Yang, X. et al. Structuralbasis for protein-protein interactions in the 14-3-3 protein family. Proceedings of the National Academy of Sciences of the United States of America 103, 17237-17242, doi:10.1073/pnas.0605779103 (2006).

23 Rajakulendran, T., Sahmi, M., Lefrancois, M., Sicheri, F. \& Therrien, M. A dimerizationdependent mechanism drives RAF catalytic activation. Nature 461, 542-545, doi:10.1038/nature08314 (2009).

24 McClendon, C. L., Kornev, A. P., Gilson, M. K. \& Taylor, S. S. Dynamic architecture of a protein kinase. Proceedings of the National Academy of Sciences of the United States of America 111, E4623-4631, doi:10.1073/pnas.1418402111 (2014). 
25 Thevakumaran, N. et al. Crystal structure of a BRAF kinase domain monomer explains basis for allosteric regulation. Nat Struct Mol Biol 22, 37-43, doi:10.1038/nsmb.2924 (2015).

26 Peng, S. B. et al. Inhibition of RAF Isoforms and Active Dimers by LY3009120 Leads to Anti-tumor Activities in RAS or BRAF Mutant Cancers. Cancer Cell 28, 384-398, doi:10.1016/j.ccell.2015.08.002(2015).

27 Karoulia, Z. et al. An Integrated Model of RAF Inhibitor Action Predicts Inhibitor Activity against Oncogenic BRAF Signaling. Cancer Cell 30, 485-498, doi:10.1016/j.ccell.2016.06.024 (2016).

28 Fischmann, T. O. et al. Crystal structures of MEK1 binary and ternary complexes with nucleotides and inhibitors. Biochemistry 48, 2661-2674, doi:10.1021/bi801898e (2009).

29 Nassar, N. et al. The 2.2 A crystal structure of the Ras-binding domain of the serine/threonine kinase c-Raf1 in complex with Rap1A and a GTP analogue. Nature 375, 554-560, doi:10.1038/375554a0 (1995).

30 Herrmann, C., Martin, G. A. \& Wittinghofer, A. Quantitative analysis of the complex between p21ras and the Ras-binding domain of the human Raf-1 protein kinase. $J$ Biol Chem 270, 2901-2905. (1995).

31 Fetics, S. K. et al. Allosteric effects of the oncogenic RasQ61L mutant on Raf-RBD. Structure 23, 505-516, doi:10.1016/j.str.2014.12.017 (2015).

32 Tran, T. H. et al. KRAS interaction with RAF1 RAS-binding domain and cysteine-rich domain provides insights into RAS-mediated RAF activation. Nat Commun 12, 1176, doi:10.1038/s41467-021-21422-x (2021).

33 Terrell, E. M. et al. Distinct Binding Preferences between Ras and Raf Family Members and the Impact on Oncogenic Ras Signaling. Mol Cell 76, 872-884 e875, doi:10.1016/j.molcel.2019.09.004 (2019).

34 Haling, J. R. et al. Structure of the BRAF-MEK complex reveals a kinase activity independent role for BRAF in MAPK signaling. Cancer Cell 26, 402-413, doi:10.1016/j.ccr.2014.07.007 (2014).

35 Cookis, T. \& Mattos, C. Crystal structure reveals the full Ras-Raf interface and advances mechanistic understanding of Raf activation. Biomolecules 11, 996, doi:10.3390/biom11070996(2021).

36 Mastronarde, D. N. Automated electron microscope tomography using robust prediction of specimen movements. J Struct Biol 152, 36-51, doi:10.1016/j.jsb.2005.07.007 (2005).

37 Zivanov, J. et al. New tools for automated high-resolution cryo-EM structure determination in RELION-3. Elife 7, doi:10.7554/eLife.42166 (2018).

38 Zheng, S. Q. et al. MotionCor2: anisotropic correction of beam-induced motion for improved cryo-electron microscopy. Nat Methods 14, 331-332, doi:10.1038/nmeth.4193 (2017).

39 Zhang, K. Getf: Real-time CTF determination and correction. J Struct Biol 193, 1-12, doi:10.1016/j.jsb.2015.11.003 (2016).

40 Athuluri-Divakar, S. K. et al. A Small Molecule RAS-Mimetic Disrupts RAS Association with Effector Proteins to Block Signaling. Cell 165, 643-655, doi:10.1016/j.cell.2016.03.045 (2016).

41 Mott, H. R. et al. The solution structure of the Raf-1 cysteine-rich domain: a novel ras and phospholipid binding site. Proceedings of the National Academy of Sciences of the United States of America 93, 8312-8317 (1996). 
42 Lito, P. et al. Disruption of CRAF-mediated MEK activation is required for effective MEK inhibition in KRAS mutant tumors. Cancer Cell 25, 697-710, doi:10.1016/j.ccr.2014.03.011(2014).

43 King, A. J. et al. Demonstration of a genetic therapeutic index for tumors expressing oncogenic BRAF by the kinase inhibitor SB-590885. Cancer Res 66, 11100-11105, doi:10.1158/0008-5472.Can-06-2554 (2006).

44 Molzan, M. \& Ottmann, C. Synergistic binding of the phosphorylated S233 - and S259binding sites of C-RAF to one 14-3-3 $\zeta$ dimer. $J$ Mol Biol 423, 486-495, doi:10.1016/j.jmb.2012.08.009(2012).

45 Pettersen, E. F. et al. UCSF Chimera--a visualization system for exploratory research and analysis. J Comput Chem 25, 1605-1612, doi:10.1002/jcc.20084 (2004).

46 Emsley, P. \& Cowtan, K. Coot: model-building tools for molecular graphics. Acta Crystallogr D Biol Crystallogr 60, 2126-2132, doi:10.1107/s0907444904019158 (2004).

47 Adams, P. D. et al. PHENIX: a comprehensive Python-based system for macromolecular structure solution. Acta Crystallogr D Biol Crystallogr 66, 213-221, doi:10.1107/s0907444909052925 (2010).

\section{Acknowledgements}

This research was supported by Federal funds from the National Cancer Institute, National Institutes of Health, under project numbers ZIA BC 010329 (D.K.M) and ZIA BC 011744 (P.Z.). This work utilized the NCI/NICE cryo-EM Facility, and we would like to thank Dr. Rick Huang for help with cryo-EM data collection. The authors also thank Dr. Dan Shi and Dr. Dewight Williams for their assistance in collecting diagnostic cryo-EM data at the NCI-Frederick cryo-EM Facility and at the John M. Cowley Center for High Resolution Electron Microscopy, Arizona State University, respectively and for their helpful discussions. In addition, we thank Dr. Ming Zhou (Inova Schar Cancer Institute) for mass spectrometry analysis of the BRAF complexes, Elizabeth Terrell for technical assistance in performing the RAS/RAF BRET assays, and Dr. Dominic Esposito (FNLCR Protein Expression Laboratory) for the KRAS protein used in fluorescent polarization assays. This study utilized the computational resources of the HighPerformance Computing Biowulf cluster of the NIH (http://hpc.nih.gov).

\section{Author Contributions}

J.A.M.F., D.E.D., D.K.M., P.Z. conceived the project, interpreted the results, and wrote the paper. D.E.D. expressed and affinity purified all BRAF complexes. J.A.M.F. further purified all 
BRAF complexes by size exclusion chromatography and performed fluorescence polarization assays. D.E.D. performed mutagenesis, co-immunoprecipitation, pull-down, and BRET assays. J.A.M.F. screen, optimized, prepare cryo-grid samples, and collected electron microscopy data. J.A.M.F. and P.Z. processed, built, and analyzed Cryo-EM models. D.K.M. and P.Z. provided funding resources and supervision.

\section{Competing Interests}

The authors declare no competing interests. 


\section{Figure 1}

a

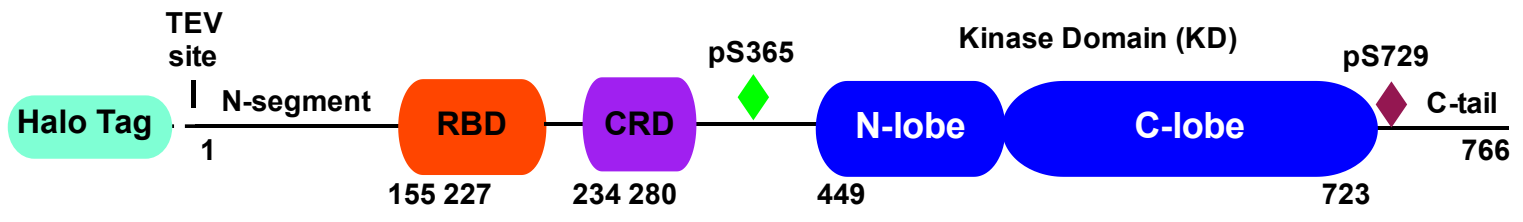

b
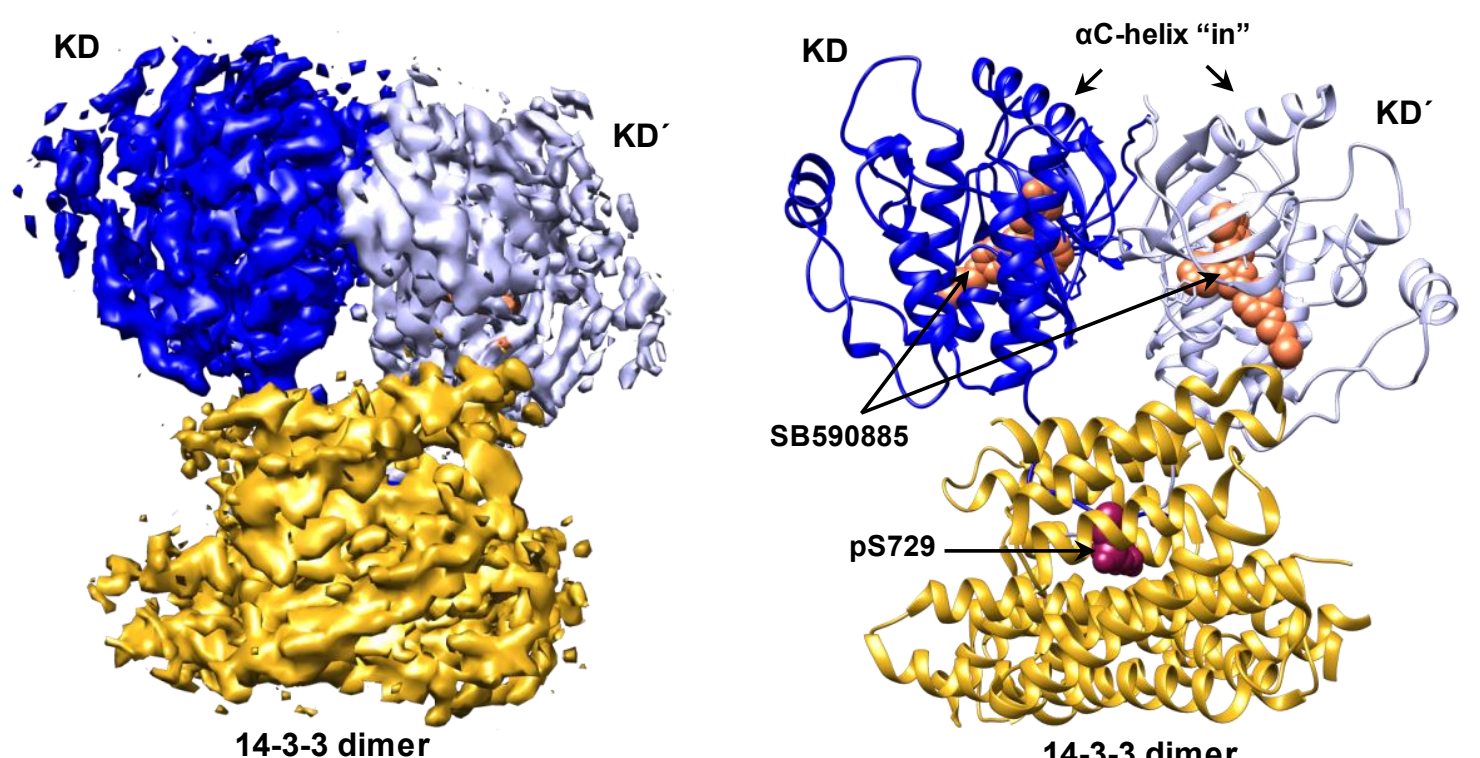

14-3-3 dimer

C

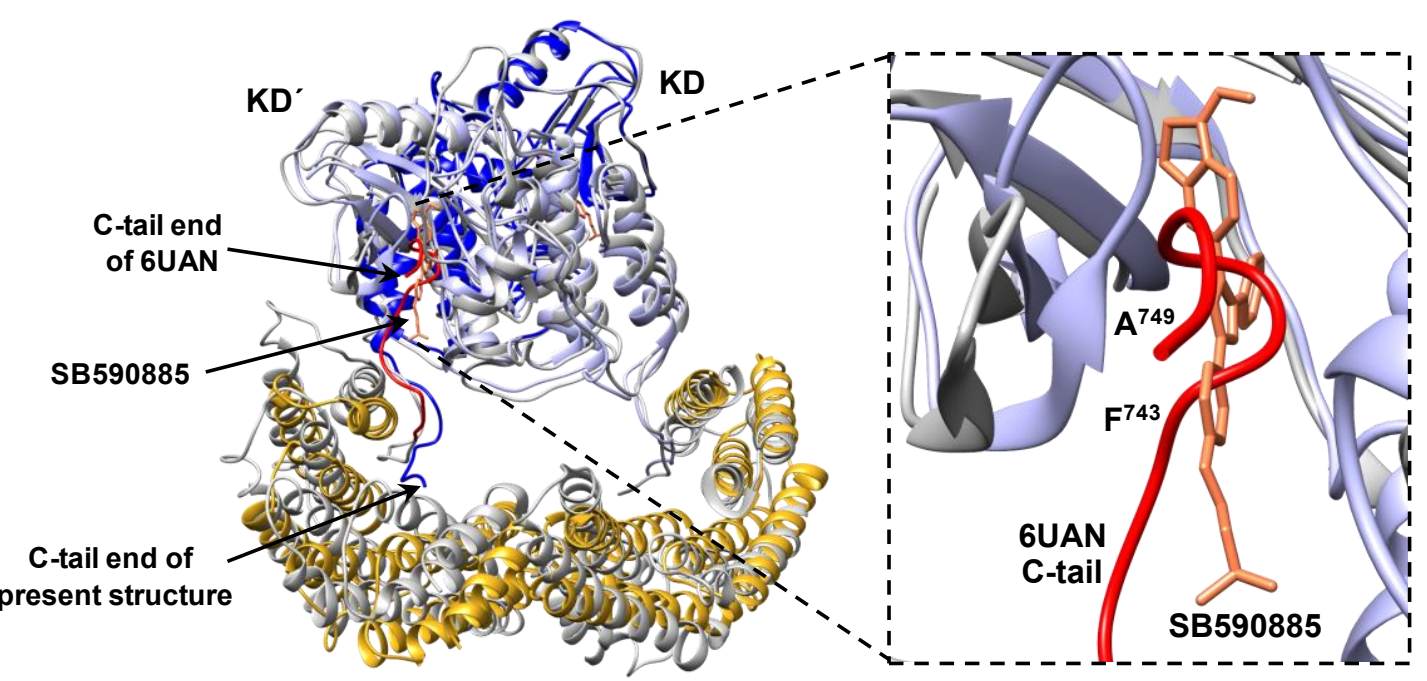

14-3-3 dimer 
Figure 2

a
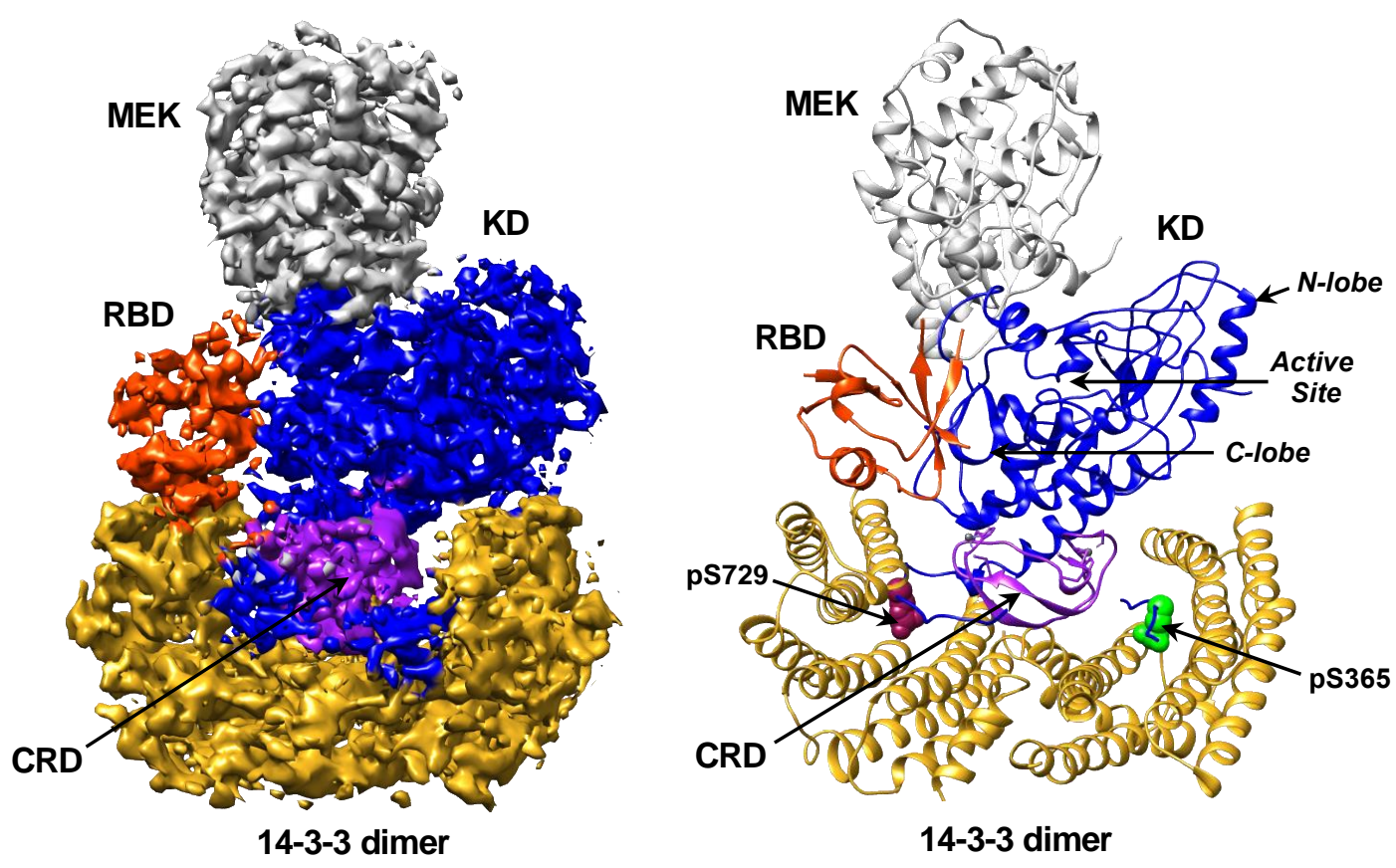

b

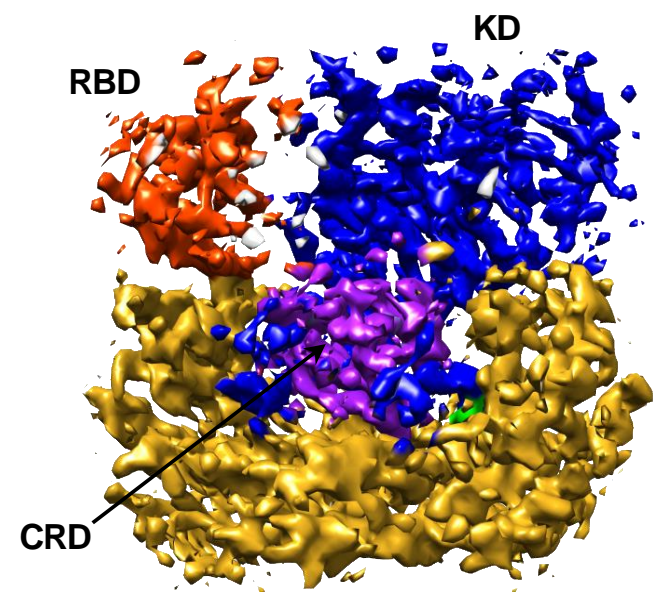

14-3-3 dimer

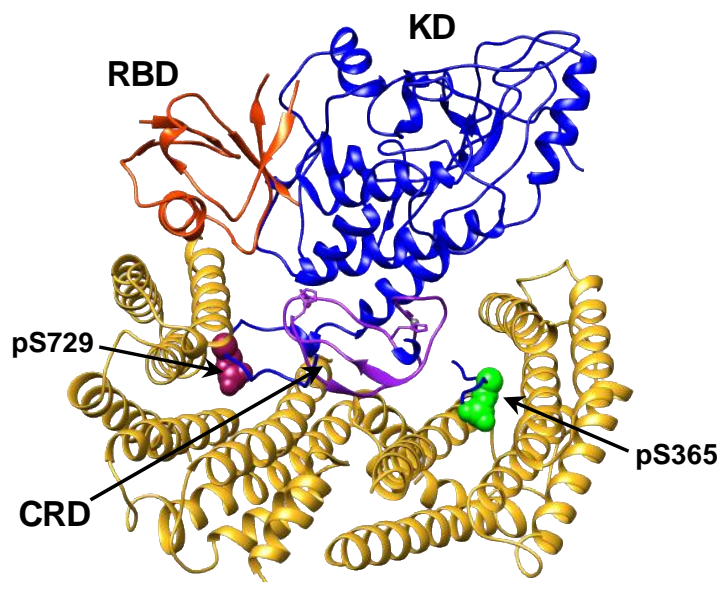

14-3-3 dimer 


\section{Figure 3}

a
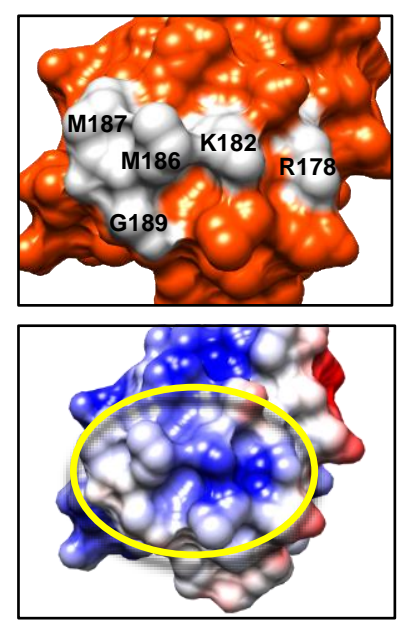

C

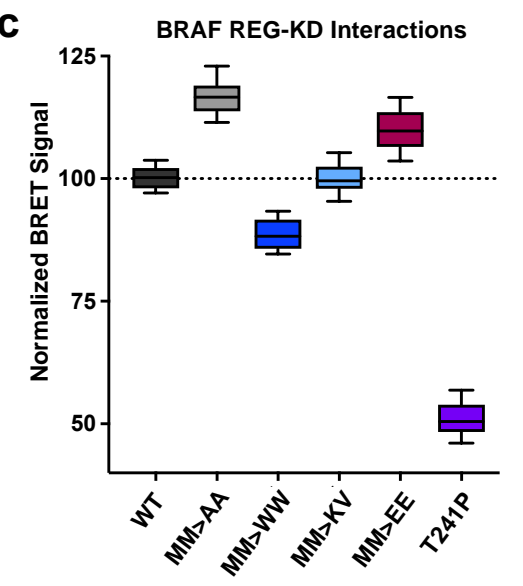

14-3-3
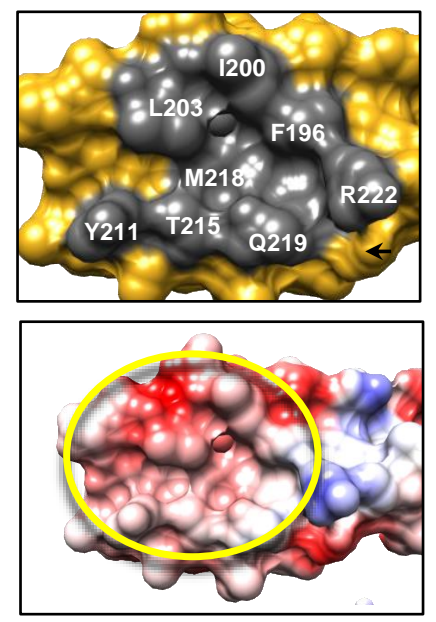

b

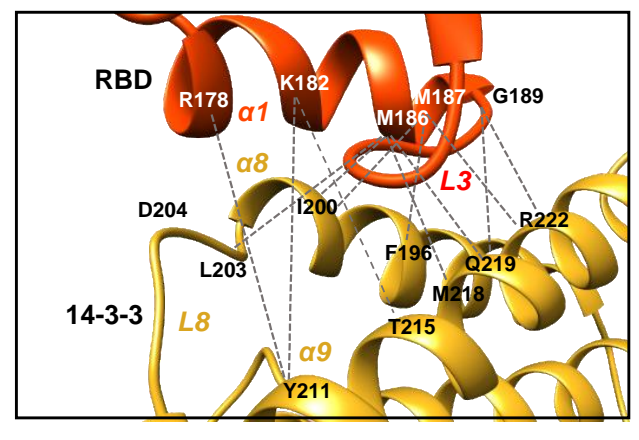

a1

L3

BRAF 178 RDSLKKALMMRGLIPECC 195

ARAF 42 YDSLDKALKVRGLNQDCC 59

CRAF 79 HDCLMKALKVRGLQPECC 96

e

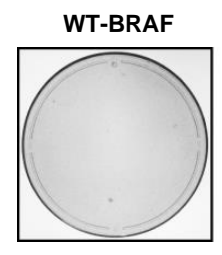

$M M>K V$

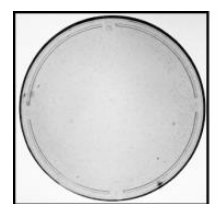

d

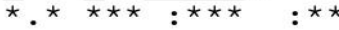

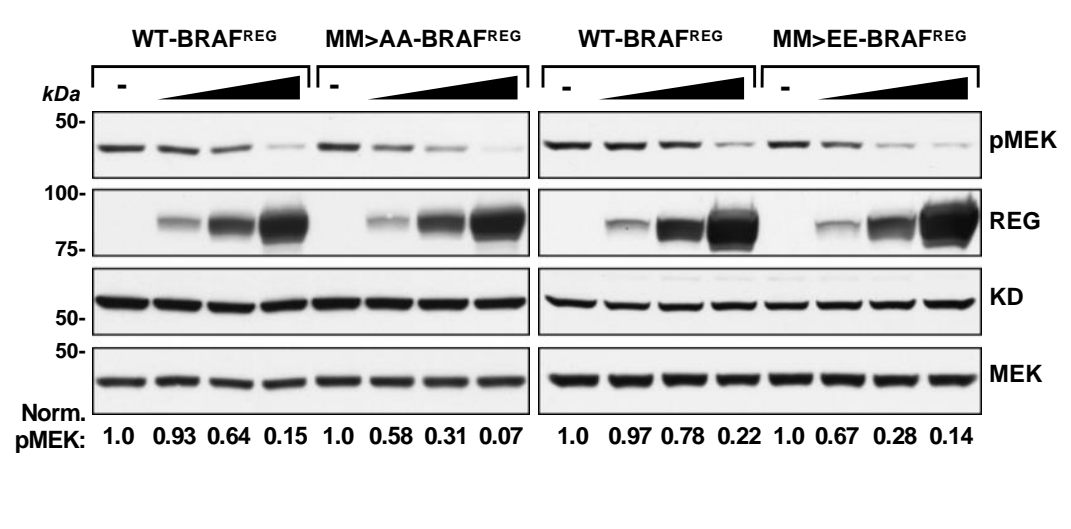

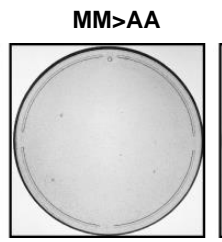

$M M>E E$

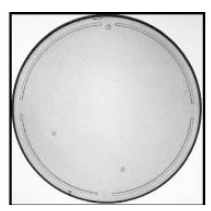

$M M>W W$

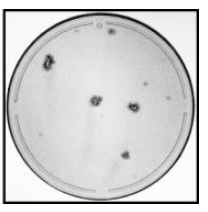

T241P

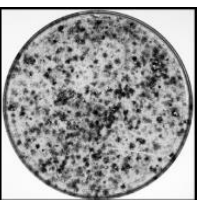

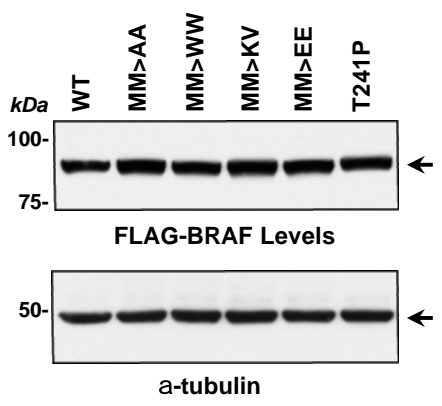


Figure 4

a

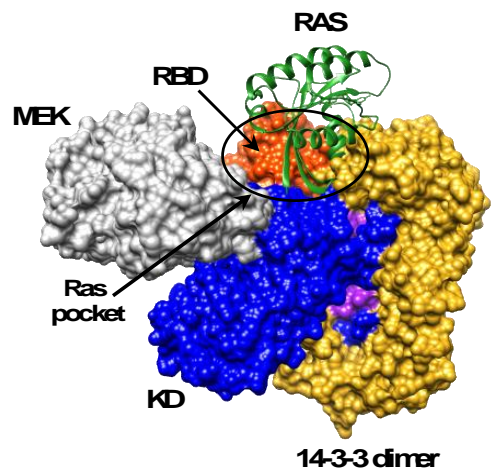

C

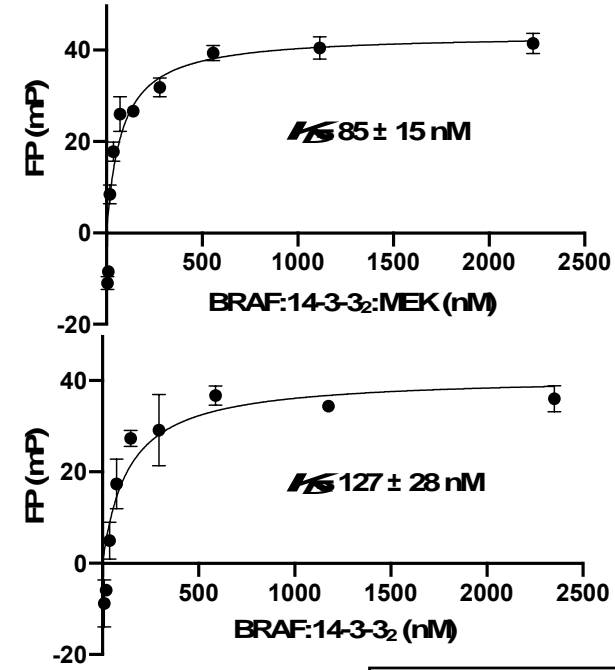

b

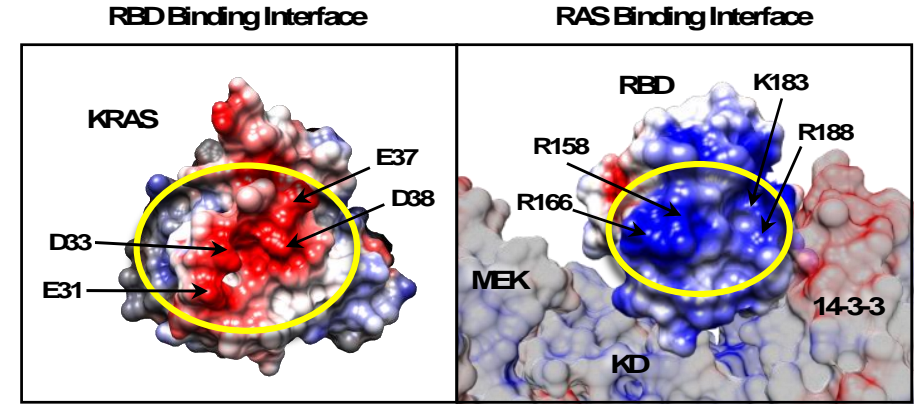

d

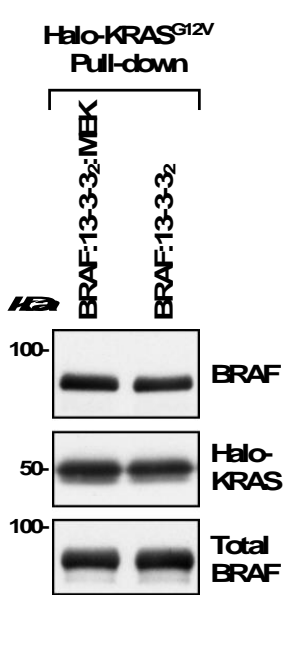

$\mathbf{e}$

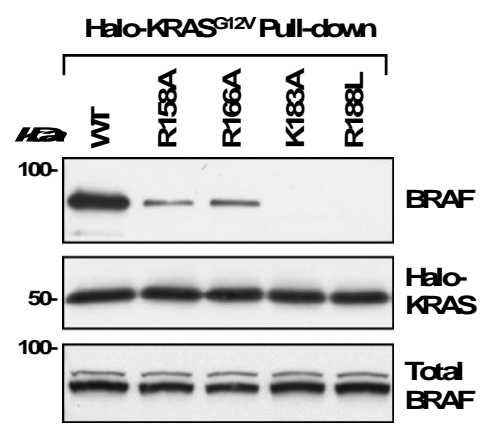

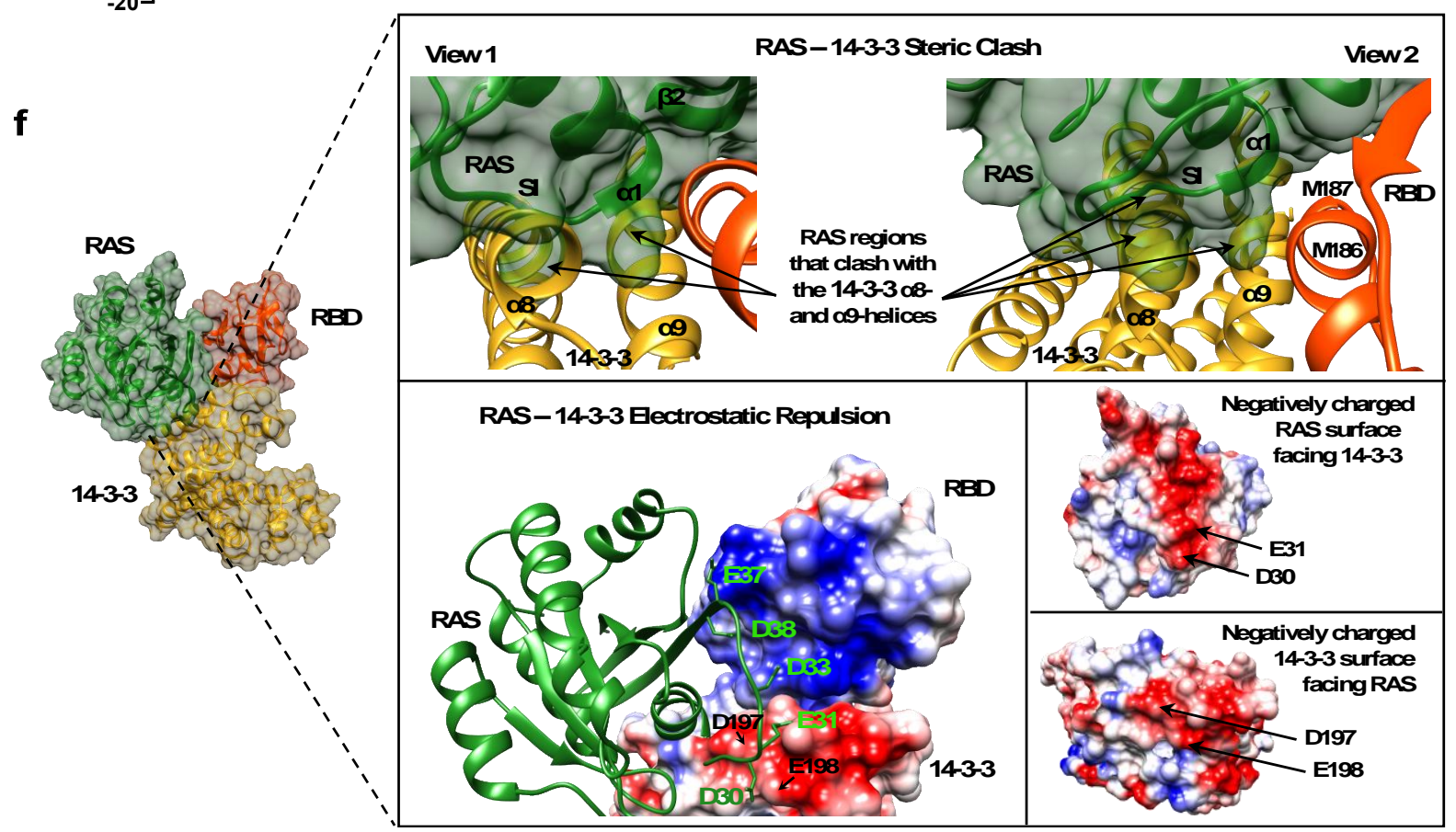




\section{Figure 5}

a

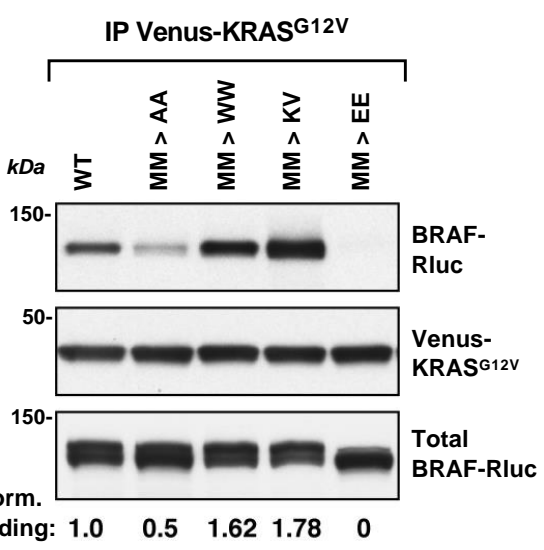

C

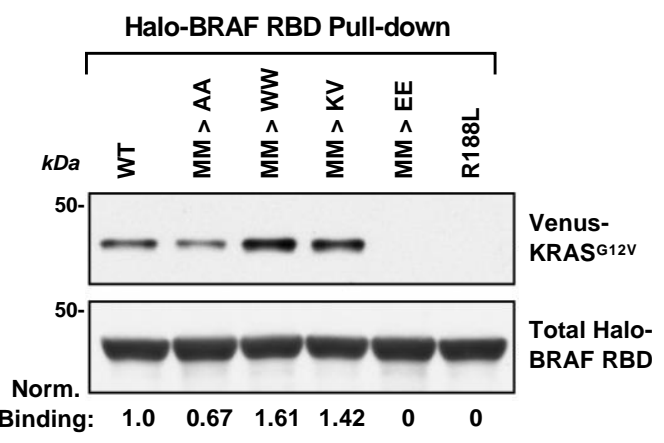

b

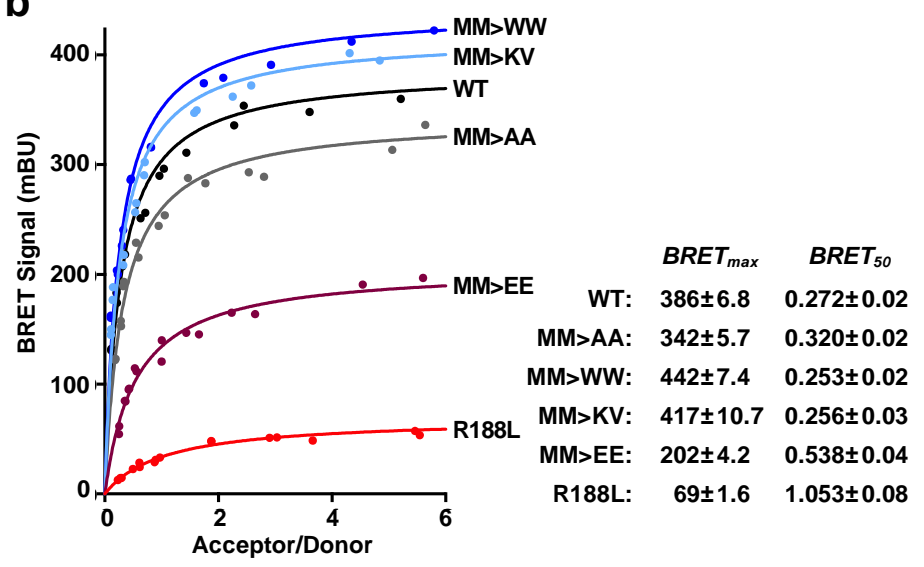

d

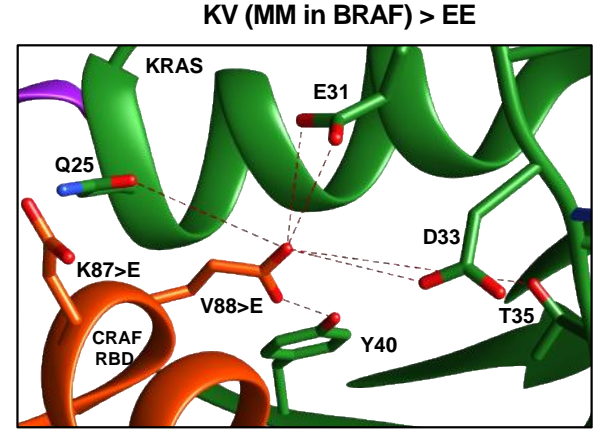

e

WT CRAF RBD

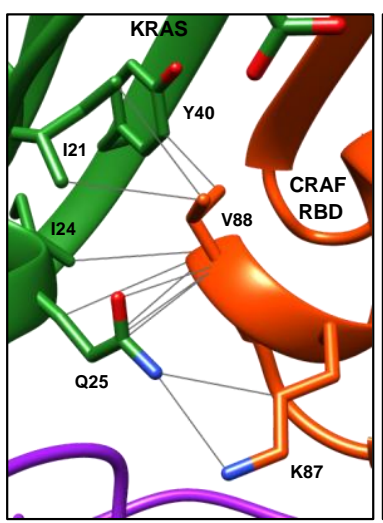

KV (MM in BRAF) > WW

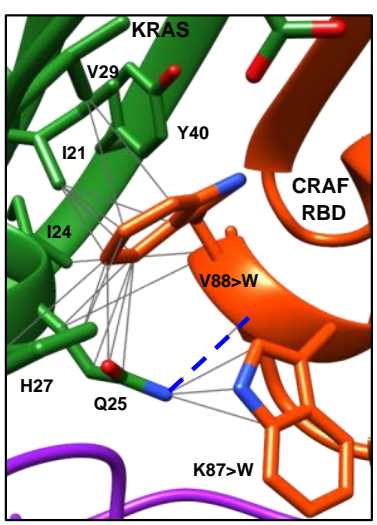

KV $(M M$ in BRAF) $>$ MM

KV (MM in BRAF) > AA

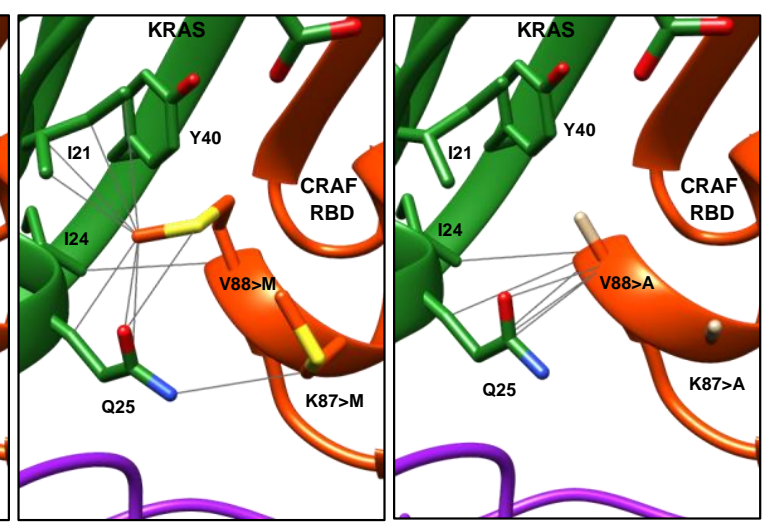


Figure 6

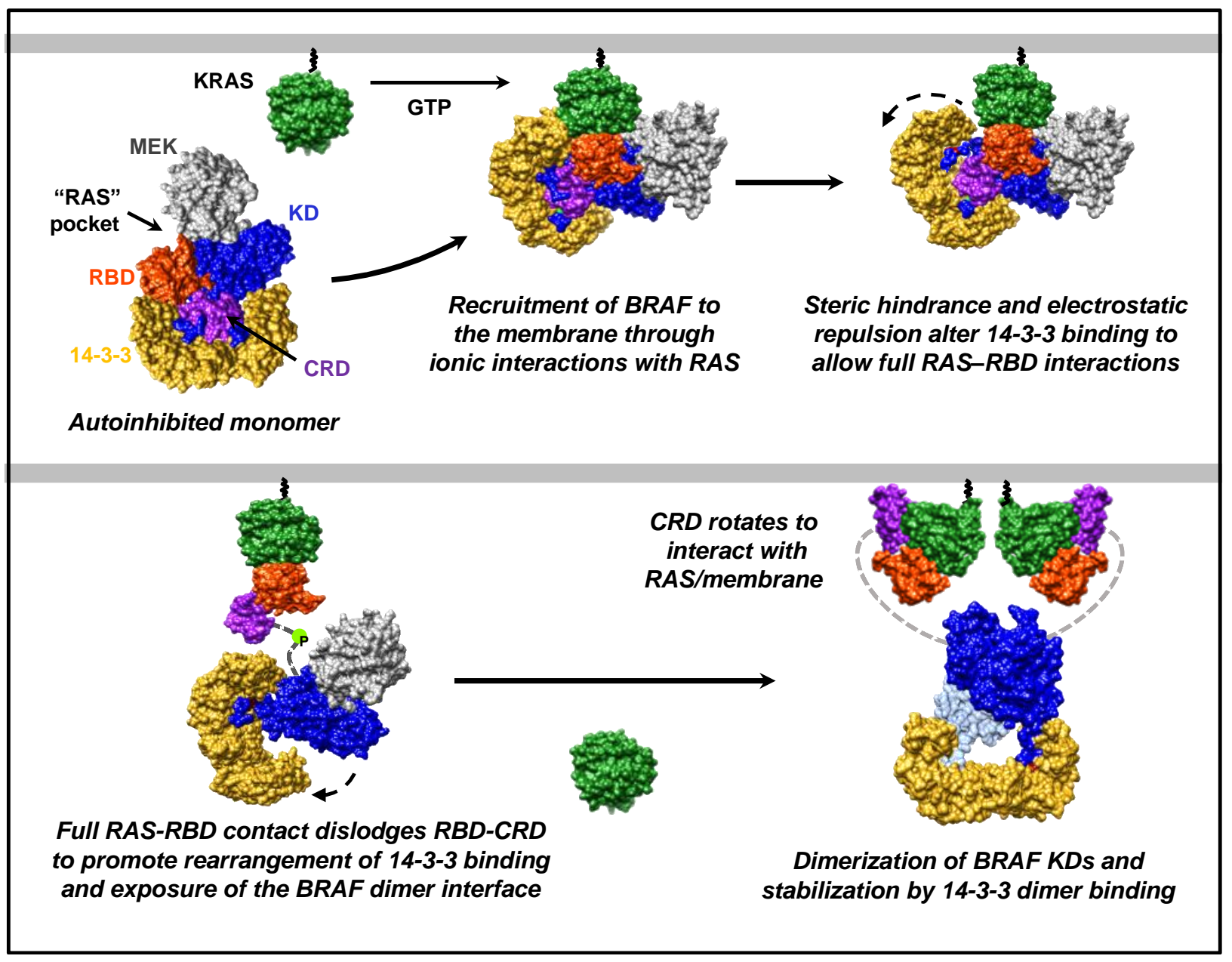




\section{Figure Legends}

\section{Fig. 1. Cryo-EM structure of inhibitor-bound $\mathrm{BRAF}_{2}: 14-3-3_{2}$ dimer complex}

a BRAF domain organization is shown with color coding: RAS binding domain (RBD, red orange), cysteine-rich domain (CRD, purple), and the kinase domain (KD, blue). BRAF residues S365 (green) and S729 (dark red) serve as 14-3-3 binding sites when phosphorylated. b Cryo-EM density map at $3.9 \AA$ A resolution (left) and structure (right) of the $\mathrm{BRAF}_{2}: 14-3-3_{2}$ complex. BRAF domains are colored as in $\mathbf{a}$, with one $\mathrm{KD}$ protomer in light blue and the other in dark blue. SB590885 is shown in coral and the 14-3-3 dimer is colored gold. c Superposition of the SB590885-liganded BRAF $2: 14-3-3_{2}$ structure (colored as in b) with the previously reported cryoEM structure of the unliganded $\mathrm{BRAF}_{2}: 14-3-3_{2}$ complex in gray (PDB ID: 6UAN) is shown with a C $\alpha$ RMSD of $1.54 \AA$ for the overall structure. The inset demonstrates that residues F743-A749 in the C-tail end (in red) of the unliganded complex (PDB ID: 6UAN) and the BRAF inhibitor SB590885 (in coral) of the present structure occupy overlapping positions in the active site.

Fig. 2. Structures of the autoinhibited, monomeric BRAF:14-3-32:MEK and BRAF:14-3-32 complexes

a Cryo-EM density map at a 3.7 Åresolution (left) and structure of the BRAF:14-3-32:MEK (right) autoinhibited complex. b Cryo-EM density map at a $4.1 \AA$ resolution (left) and structure of the BRAF:14-3-32 (right) autoinhibited complex. Regions of the BRAF monomer and the 14-3-3 dimer are colored as previously described and MEK is shown in gray.

\section{Fig. 3. Analysis of the RBD:14-3-3 interface}

a Region of RBD:14-3-3 contact, with RBD and 14-3-3 residues at the contact interface depicted as light gray and dark gray, respectively. Interacting residues are labeled (top). Electrostatic surface representation of the RBD and 14-3-3, with blue and red representing positively and 
negatively charged areas, respectively. Regions of contact between the RBD and 14-3-3 lay within the yellow circles (bottom). b The 14-3-3 $\alpha 8$-helix, $\alpha 9$-helix, and the connecting loop 8 contact the $\alpha 1$-helix and loop 3 of the B-Raf RBD. The RBD is colored in orange, 14-3-3 in gold and the interacting residues are labeled (top). Sequence alignment of RBD residues in the $\alpha 1$-helix and loop 3 of human BRAF, CRAF and ARAF (bottom). Residues at the RBD:14-3-3 interface are denoted by the shaded gray box, and identically conserved residues are shown in red. Symbols under the alignment denote the degree of conservation as follows: $(*)$ indicates positions which have a fully conserved residue, (:) indicates conservation between groups with strongly similar properties, and (.) indicates conservation between groups with weakly similar properties.

c NanoBRET assay monitoring the interaction between WT or mutant BRAFREG_Halo proteins and Nano-BRAFKD in live cells to determine the effect of the indicated mutations on the ability of BRAF to form a stable, autoinhibited complex. BRET signals (normalized to WT set at 100) of quadruplicate wells from five independent experiments were used to generate a box and whiskers plot, with the center line representing the median, the box limits extending from the $25^{\text {th }}$ to $75^{\text {th }}$ percentile, and the whiskers extending from the minimum to maximum values. dLysates of 293FT cells transiently expressing Nano-WT-BRAFKD alone or co-expressing Nano-WT-BRAFKD with increasing amounts of WT-, M186A/M187A-, or M186E/M187E-BRAFREG_Halo were examined by immunoblot analysis for WT-BRAF ${ }^{R E G}-H_{a l o}$, Nano-WT-BRAFREG, and pMEK levels. Blots are representative of three independent experiments with similar results. e NIH-3T3 cells were infected with retroviruses encoding the indicated WT or mutant FLAG-BRAFFL proteins. Three weeks post-infection, foci were visualized by methylene blue staining. Stained plates are representative of three independent experiments with similar results. Source data are provided as a Source Data file.

\section{Fig. 4. RAS, BRAF RBD, and 14-3-3 interactions}

a Superimposition of KRAS (from KRAS:CRAF_RBD structure PDB ID: 6XI7, colored in green) onto the autoinhibited BRAF:14-3-32:MEK complex is shown to demonstrate the fit of KRAS into 
the "RAS pocket" for RAS:RBD binding. b Electrostatic surface representation of the RAS:RBD binding interface (yellow circle), with critical ionic bond forming residues indicated on the surfaces of KRAS (PDB ID: 6XI7, left) and the RBD of the BRAF:14-3-32:MEK complex (right). c Binding affinities of the BRAF:14-3-32:MEK (top) and BRAF:14-3-3 (bottom) complexes to KRAS(GppNHp) were determined in fluorescence polarization assays. Data are represented as the mean of duplicate wells $\pm \mathrm{SD}$. The reported $K_{D}$ is the mean of three independent experiments. d Purified BRAF:14-3-32:MEK and BRAF:14-3-32 complexes were evaluated for their ability to bind Halo-tagged KRASG12V in pull-down assays. Blots are representative of two independent experiments with similar results. e WT or BRAF full-length proteins containing mutations in key basic RBD residues mediating ionic bond interactions with RAS were examined for binding to Halo-KRAS ${ }^{\mathrm{G} 12 \mathrm{~V}}$ in pull-down assays. Blots are representative of three independent experiments with similar results. f Superimposition of KRAS onto the autoinhibited BRAF:14-3-3 2 :MEK complex indicates potential steric clash and electrostatic repulsion between RAS and the 14-3-3 protomer at the RBD:14-3-3 interface upon full RAS:RBD contact. The steric clash (top insets) would occur between RAS $\alpha 1$-helix, switch I (SI) and $\beta 2$ sheet (ribbon and surface in green) and the $\alpha 8$ - and $\alpha 9$ - helices of 14-3-3 (ribbon in gold). In the same region, similarly charged residues in KRAS, D30 and E31, and 14-3-3, D197 and D198, would cause electrostatic repulsion (bottom inset, RAS ribbon structure in green superimposed onto the electrostatic surface representation of the RBD and 14-3-3). Source data are provided as a Source Data file.

\section{Fig. 5. RBD Residues at the 14-3-3 Interface Contribute to RAS Binding}

a WT-BRAF-Rluc and the indicated M186/M187 mutants were assessed for binding to Venus$\mathrm{KRAS}^{\mathrm{G} 12 \mathrm{~V}}$ in co-immunoprecipitation assays. Lysates were also monitored for BRAF-Rluc protein levels. Blots are representative of three independent experiments with similar results. b BRET saturation curves are shown examining the interaction of WT-,or M186/ M187 mutant BRAF-Rluc proteins with Venus-KRAS ${ }^{\mathrm{G} 12 \mathrm{~V}}$ in live cells. As a control, an RBD protein containing the R188L mutation that disrupts the RAF-RAS interaction was also evaluated. BRET $\max$ and 
BRET $_{50}$ values are listed \pm standard error on the right. Saturation curves were repeated 3 times with similar results. c Binding of Venus-KRAS ${ }^{\mathrm{G} 12 \mathrm{~V}}$ to WT or mutant Halo-BRAF RBD proteins was assessed in pulldown assays. Blots are representative of three independent experiments with similar results. d Modeling the effect of substituting the CRAF K87/V88 amino acids for K87E/V88E, which would generate electrostatic repulsion (indicated by dark red lines) with polar (Q25, T35, and Y40) and negatively charged (E31 and D33) KRAS residues. e Modeling the effect of substituting the CRAF K87/V88 amino acids for K87W/V88W, K87M/V88M, or K87A/V88A in the RBD:RAS structure (KRAS:CRAF_RBD_CRD structure PDB ID: 6XI7, colored in green for KRAS, orange for CRAF RBD and violet for CRAF CRD). Gray lines indicate potential nonelectrostatic interactions and blue dashed line show potential hydrogen bonds. Source data are provided as a Source Data file.

Fig. 6. Model for BRAF monomer to dimer transition Upon RAS activation, the autoinhibited BRAF monomer is recruited to the plasma membrane through direct ionic interactions between the BRAF RBD and the switch I region of RAS. As RAS forms the full spectrum of RBD contacts, steric clashes and electrostatic repulsion occur between RAS and 14$3-3$, resulting in conformational changes that dislodge the RBD/CRD and promote a rearrangement in 14-3-3 dimer binding, thus exposing the pS365 site and the BRAF dimer interface. The dislodged CRD rotates to interact with the membrane and RAS to further stabilize the interaction. The exposed KD can then dimerize and assume the active catalytic conformation that is stabilized by 14-3-3 dimer binding to the pS729 sites on each BRAF protomer. 


\title{
Supplementary Information
}

Structural insights into the BRAF monomer-to-dimer transition mediated by RAS binding

\author{
Juliana A. Martinez Fiesco ${ }^{1 \wedge}$, David E. Durrant ${ }^{2 \wedge}$, Deborah K. Morrison²* \\ and Ping Zhang ${ }^{*}$
}

${ }^{1}$ Center for Structural Biology and ${ }^{2}$ Laboratory of Cell and Developmental Signaling, Center for Cancer Research, National Cancer Institute-Frederick, Frederick, MD 21702

$\wedge$ These authors contributed equally to this work

* corresponding authors: morrisod@mail.nih.gov and ping.zhang@nih.gov

\section{Supplementary Information:}

Supplementary Fig. 1-6

Supplementary Tables 1-4

Supplementary References 


\section{Supplementary Figures}
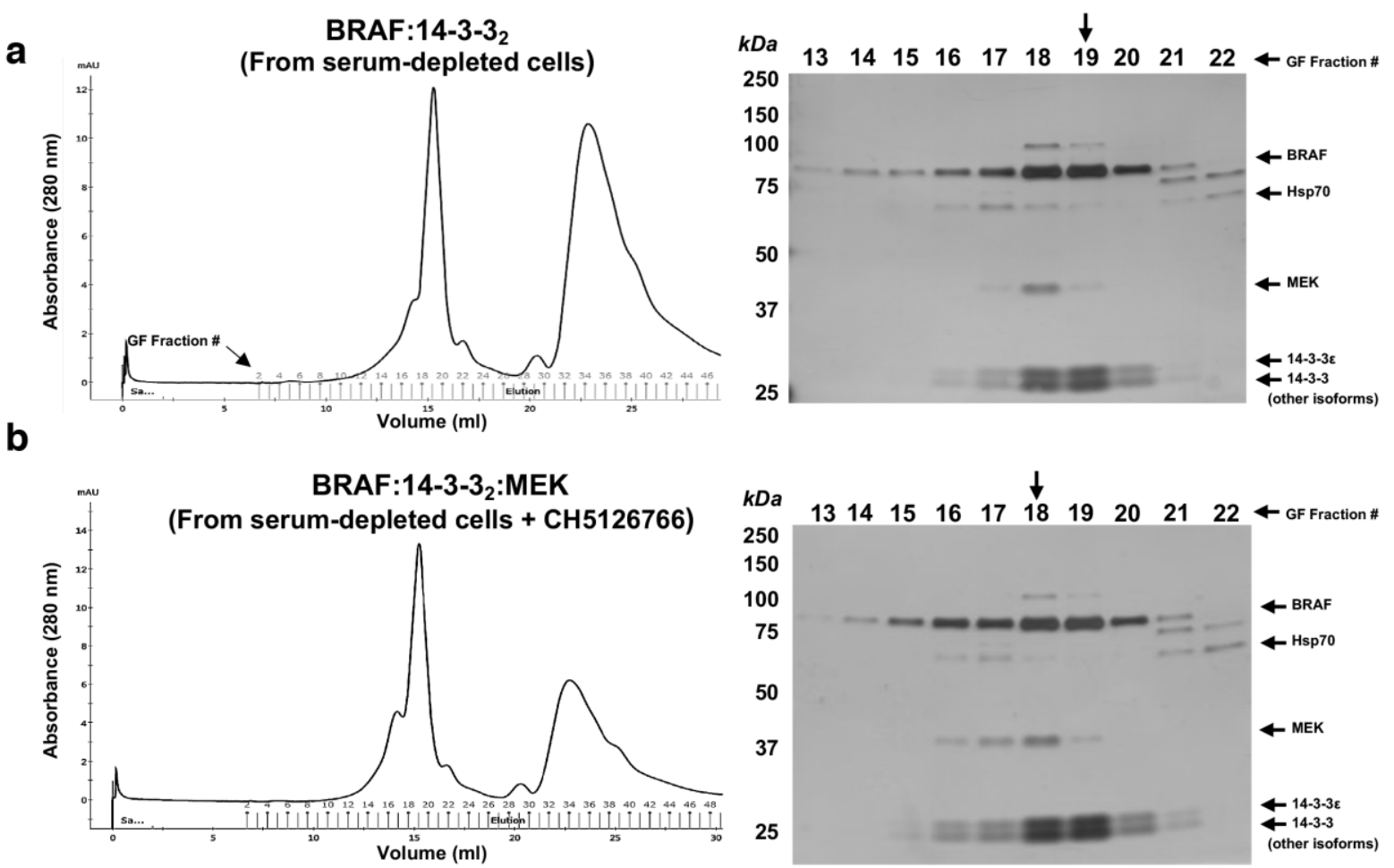

C

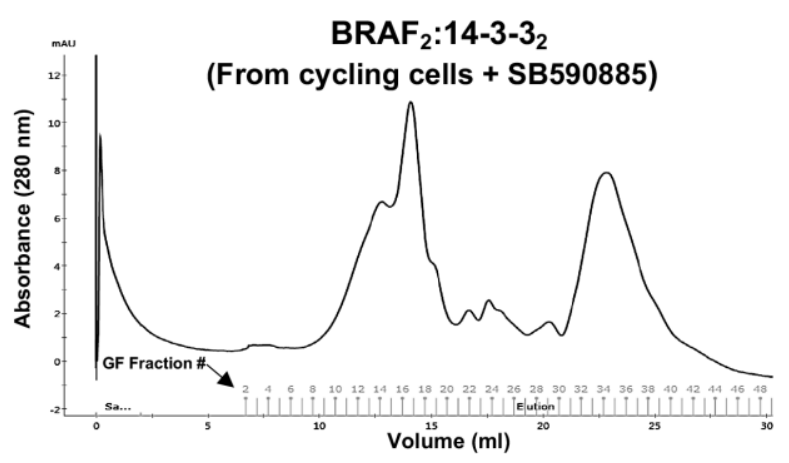

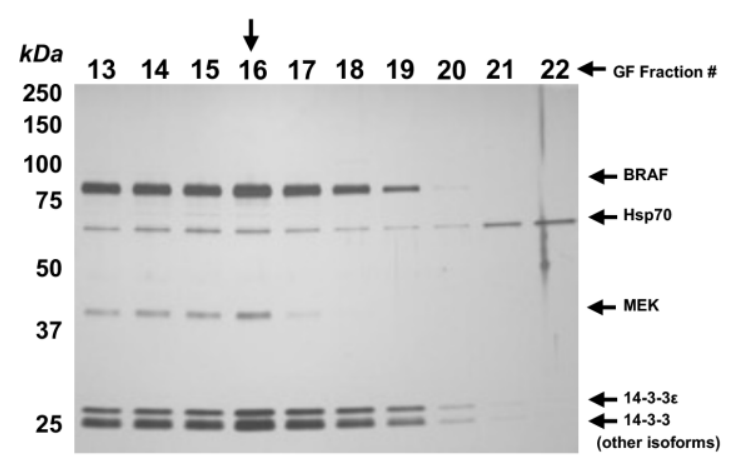

d

\begin{tabular}{|c|c|c|c|c|}
\hline Protein & Accession & BRAF:14-3-32:MEK & BRAF:14-3-32 & BRAF2:14-3-32 \\
\hline 14-3-3 protein epsilon & P62258 & 49.2 & 51.2 & 43.5 \\
\hline 14-3-3 protein zeta/delta & P63104 & 23.4 & 24.7 & 25.8 \\
\hline 14-3-3 protein beta/alpha & P31946 & 8.5 & 8.1 & 9.5 \\
\hline 14-3-3 protein eta & Q04917 & 6.1 & 6.4 & 9.6 \\
\hline 14-3-3 protein gamma & P61981 & 8.5 & 5.9 & 3.9 \\
\hline 14-3-3 protein theta & P27348 & 4.3 & 3.6 & 7 \\
\hline 14-3-3 protein sigma & P31947 & 0.2 & 0.1 & 0.7 \\
\hline MEK1 & Q02750 & 53.6 & & \\
\hline MEK2 & P36507 & 46.4 & & \\
\hline
\end{tabular}

Values represent percentage of total peptides for 14-3-3 or MEK 


\section{Supplementary Fig. 1. Isolation and characterization of mammalian BRAF complexes}

BRAF:14-3-32 (a), BRAF:14-3-32:MEK (b), and BRAF $2: 14-3-3_{2}$ (c) complexes were analyzed by gel filtration (GF) (left) and SDS-PAGE/silver staining (right). The arrows indicate the sample fractions used for cryo-EM studies. Quantification of the silver stained band densities in the sample fractions used for cryoEM studies indicates a 1:1 ratio for BRAF and the 14-3-3 dimer (with all 14-3-3 isoforms pooled). The ratio of BRAF and MEK in Fraction 19 of (a) was 1:0.04, in fraction 18 of (b) was 1:0.39, and in fraction 16 of (c) was 1:0.18. d Mass spectrometry analysis of the complexes isolated in a-c. 
a
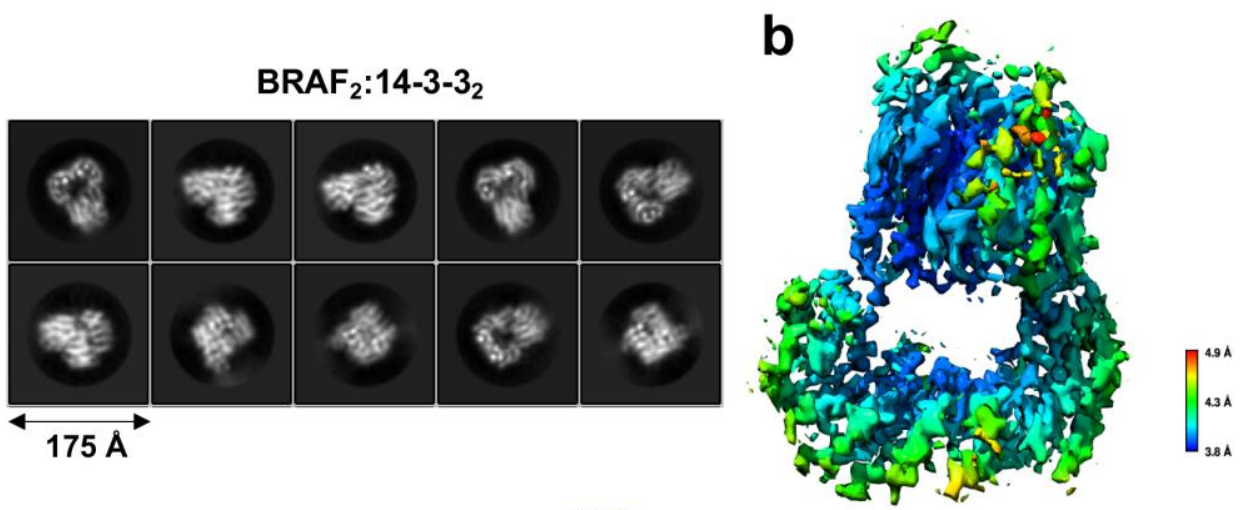

C

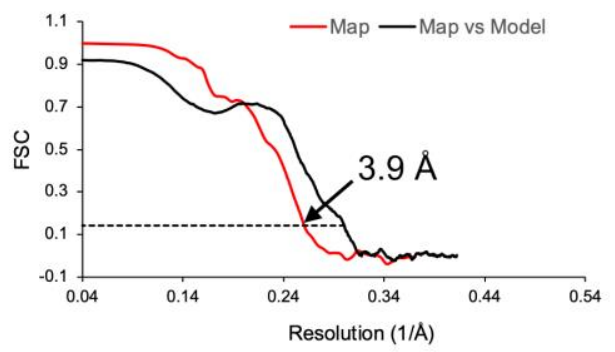

d

KDs $\alpha C$-helices

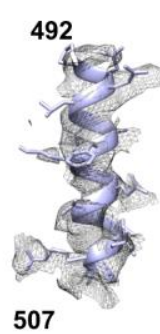

507
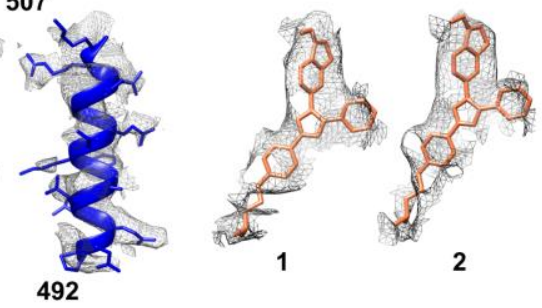

SB590885

KD'

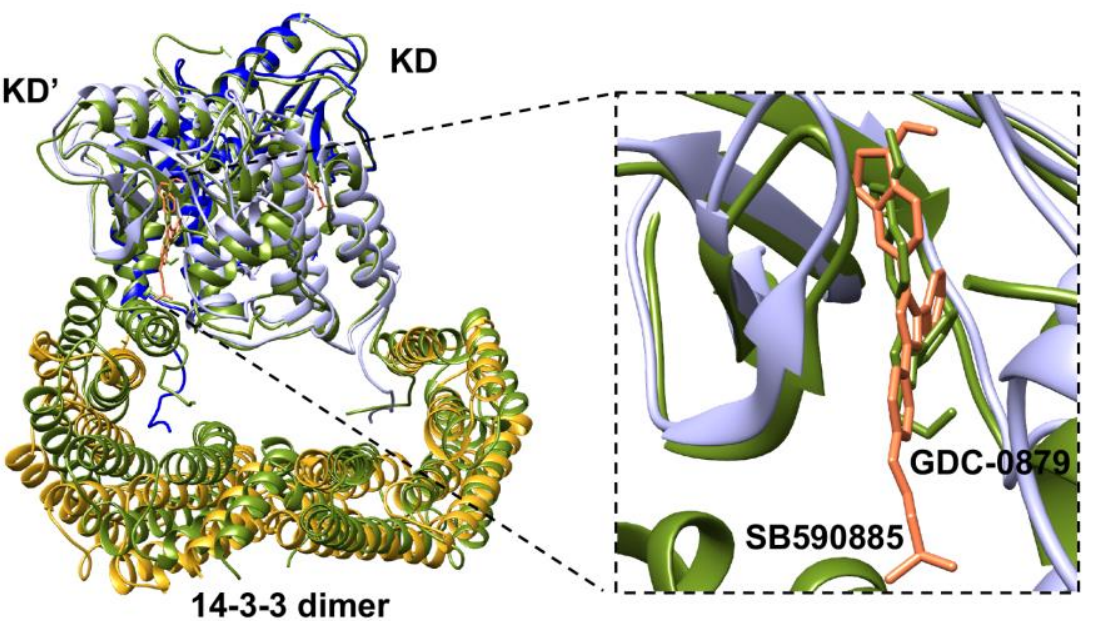




\section{Supplementary Fig. 2. Cryo-EM analysis of the $\mathrm{BRAF}_{2}: 14-3-3_{2}$ dimer complex}

a Representative cryo-EM image of 2,998 micrographs and 2D class averages of $\mathrm{BRAF}_{2}: 14-3-3_{2}$ particles. b BRAF $2: 14-3-3_{2}$ cryo-EM map colored according to local resolution. $\mathbf{c}$ FSC curves of the cryo-EM density map (left). Reported resolutions were based on the FSC $=0.143$ criteria. Red curve is the $\mathrm{BRAF}_{2}: 14-3-3_{2}$ half map and black curve is the model versus map FSC. Euler angle distribution of particles used for the global reconstruction map (right). d Cryo-EM densities corresponding to the $\alpha \mathrm{C}$-helix and the bound molecule of SB590885 in each KD protomer. e Conformation of the KDs in the liganded $\mathrm{BRAF}_{2}: 14-3-3_{2}$ complex. The KD is shown in gray with the $\alpha \mathrm{C}$-helix (blue) in the active 'in' position and the R-spine residues (pink) aligned. $\mathbf{f}$ Superposition of the SB590885-liganded BRAF $2: 14-3-3_{2}$ structure (colored as in Fig. 1) with the crystal structure of (GDC-0879)-bound BRAF KD 2 (residues D432-R735):14-3-32 (in green, PDB ID: 6U2H) is shown, with an overall Ca RMSD of $2.29 \AA$. The inset shows the SB590885 and GDC-0879 ligands occupy overlapping positions in the active site. 
a

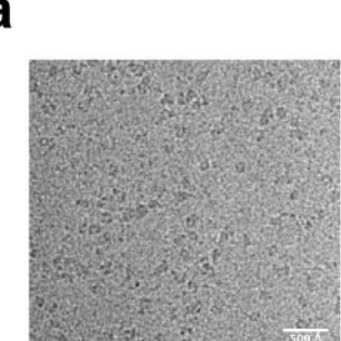

b
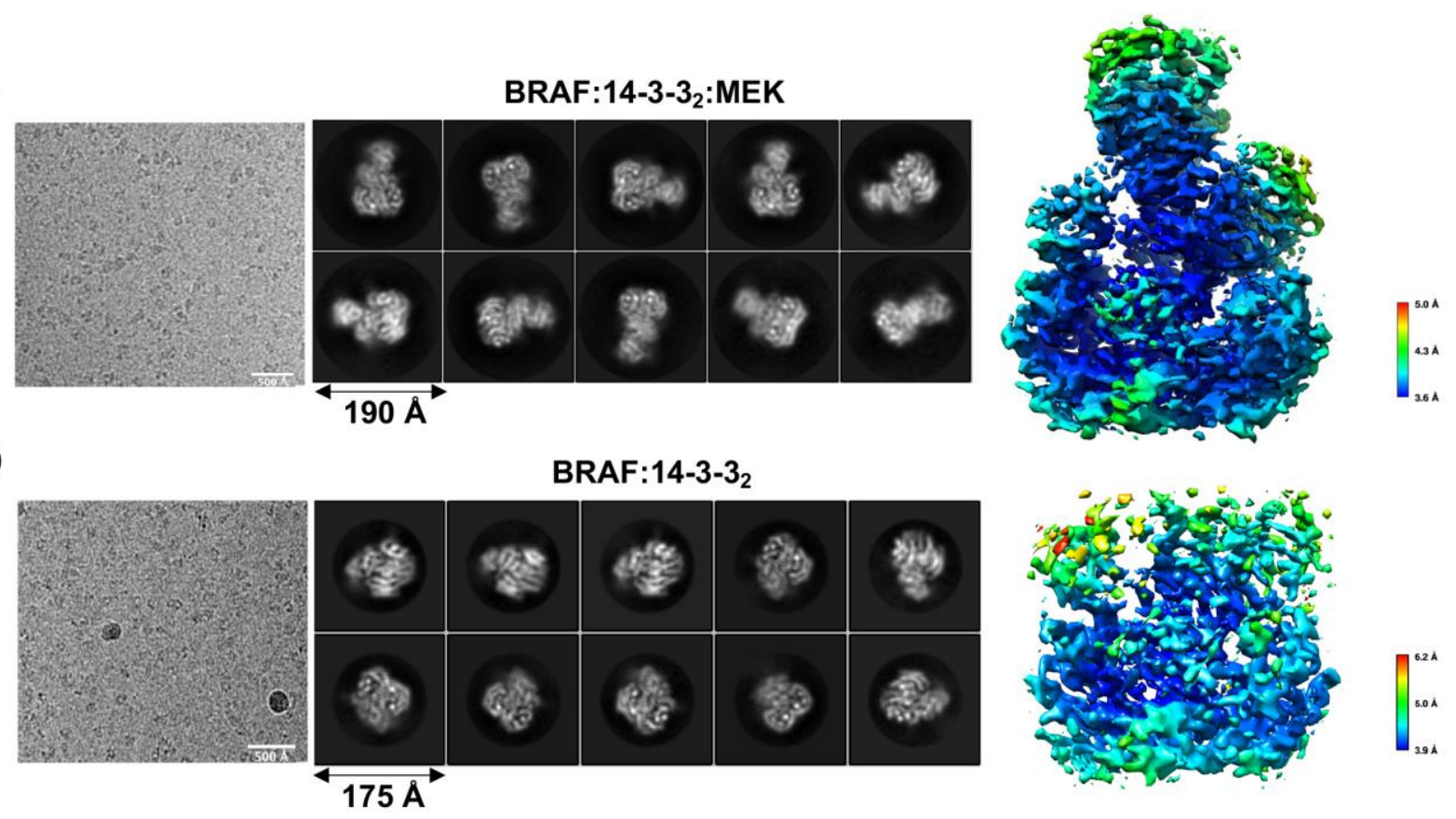

C

BRAF:14-3-3: $:$ MEK
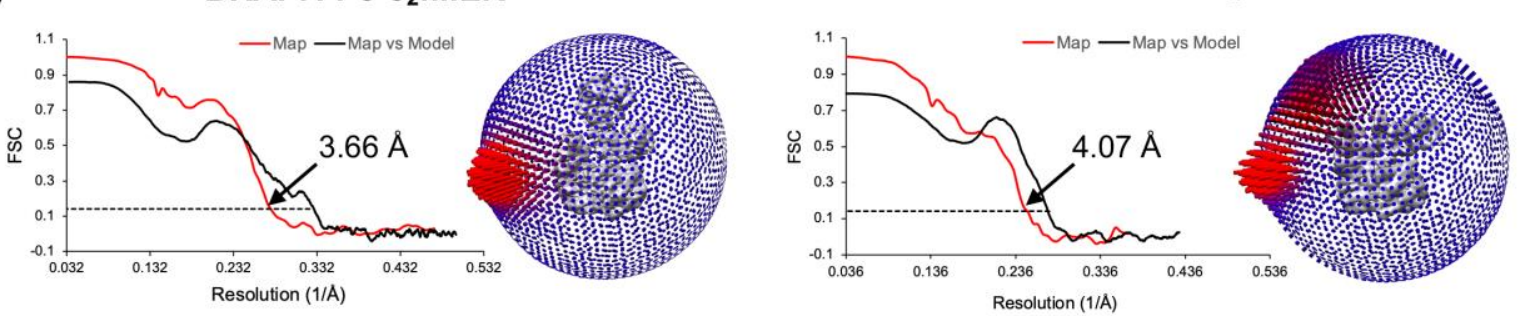

Supplementary Fig. 3. Cryo-EM analysis of autoinhibited BRAF:14-3-32:MEK and

\section{BRAF:14-3-3 2 complexes}

$\mathbf{a}$ and $\mathbf{b}$ Representative cryo-EM image and 2D class averages (left) and the cryo-EM map colored according to local resolution (right) of the BRAF:14-3-32:MEK (a) and BRAF:14-3-3 $(\mathbf{b}$ ) complexes. The complete cryo-EM data set consisted of 3,976 and 5,995 micrographs for BRAF:14-3-32:MEK and BRAF:14-3-3, respectively. c FSC curves of the cryo-EM density map and euler angle distribution of particles used for the global reconstruction map of BRAF:14-3$3_{2}:$ MEK (left) and BRAF:14-3-3 2 (right). 


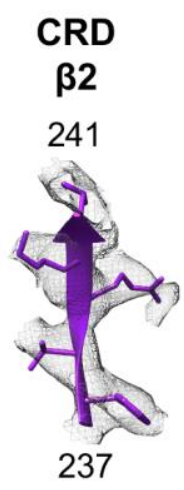

14-3-3:pS365

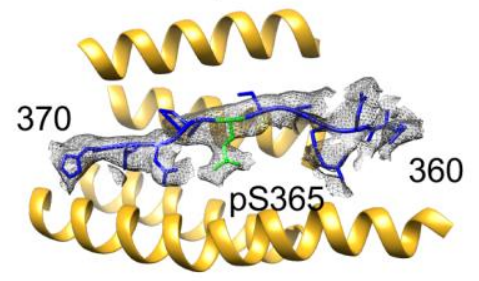

BRAF

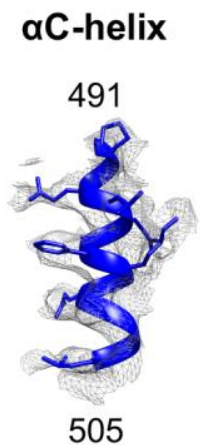

Inhibitory turn

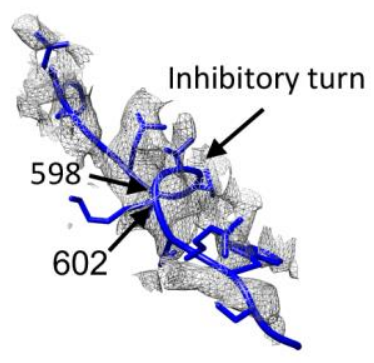

14-3-3:pS729

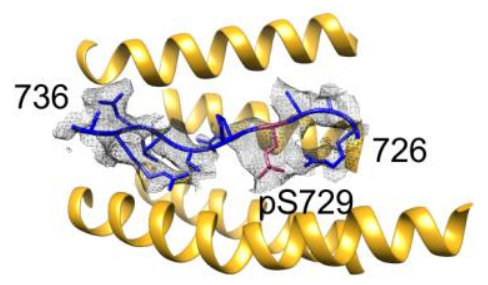

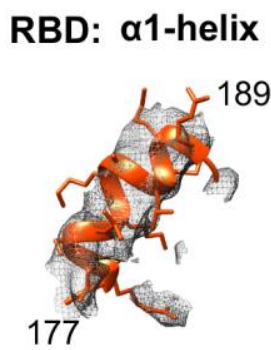
177

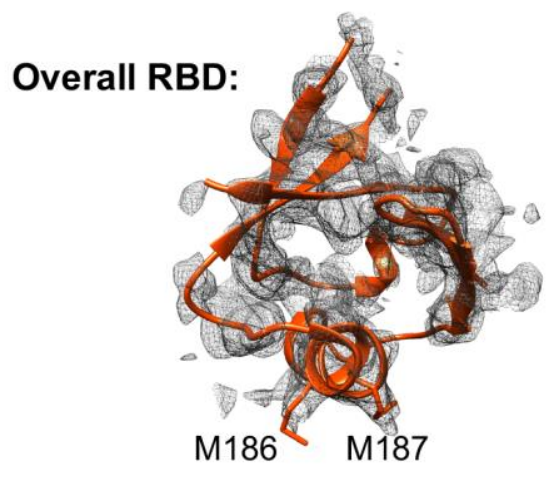

View 1

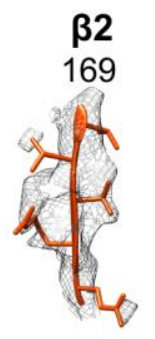

165

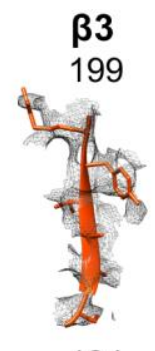

194

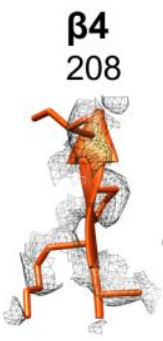

205
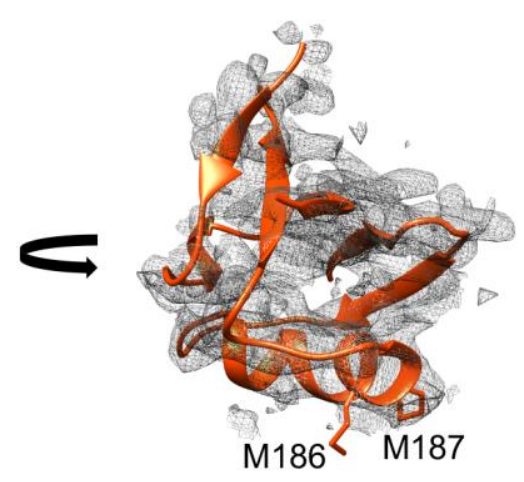

View 2

\section{Supplementary Fig. 4. Structural analysis of the BRAF:14-3-32:MEK complex}

Density maps of specific regions of BRAF (RBD, CRD, and KD), MEK, and 14-3-3 proteins are shown. Also shown, are density maps of the overall RBD, with methione residues 186 and 187 indicated. 

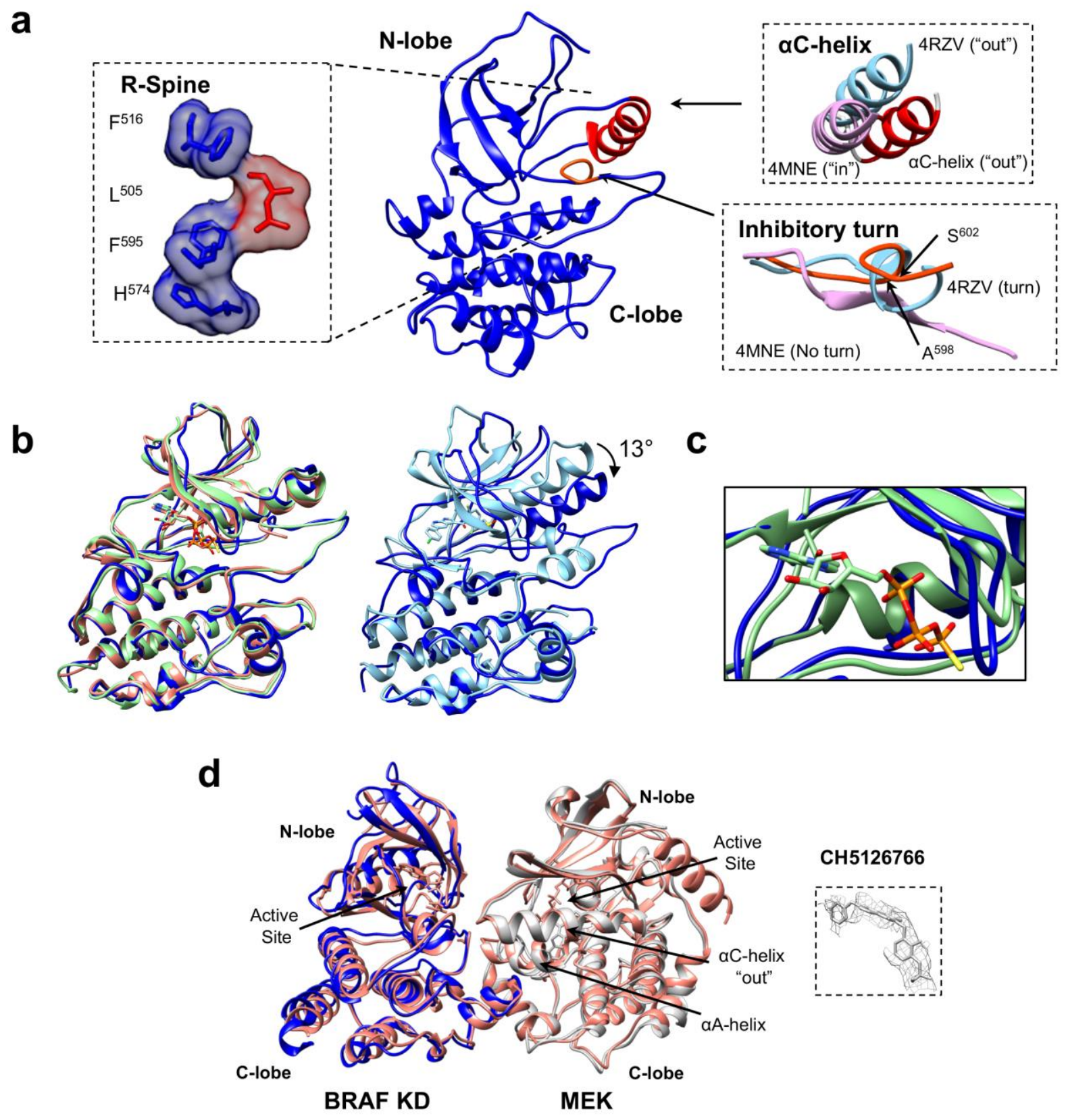

Supplementary Fig. 5. Structural analysis of the BRAF KD in the autoinhibited BRAF:14-

\section{3-32:MEK complex}

a The BRAF KD in the BRAF:14-3-32:MEK complex assumes the inactive conformation, with the aC-helix in the "out" position and the R-spine broken (left inset). Insets on the right show the comparison of specific regions of the present KD structure with published BRAF KD structures in the active (PDB ID 4MNE, in pink) and inactive, inhibitory-bound (PDB ID: 4RZV, bound to the 
RAF inhibitor vemurafenib, in blue) conformations. b (left) Superimposition of the BRAF KD (blue) in the present structure with BRAF KDs of the previously reported cryo-EM BRAF(ATP$\gamma \mathrm{S}): 14-3-3_{2}:$ MEK1 structure (with a Ca R.M.S.D. of $0.90 \AA$, PDB ID:6NYB, green), and the BRAF KD(AMP-PCP):MEK1 structure (with a C $\alpha$ R.M.S.D. of $0.82 \AA$, PDB ID: 6U2G, salmon), demonstrating the compact configuration of the $\mathrm{N}$ - and $\mathrm{C}$-lobes in these inactive $\mathrm{KD}$ structures. (right) Superimposition of the BRAF KD (blue) in the present structure with the vemurafenibbound BRAF KD structure (with a C $\alpha$ R.M.S.D. of $0.84 \AA$, PDB ID: 4RZV, light blue), which is an example of RAF inhibitor-bound BRAF KD structures exhibiting a more open $\mathrm{N}$ - and C-lobe configuration. Superimposition of these structures was done based on the C-lobe of the KD. c Superimposition of the ATP binding pockets in the present BRAF:14-3-32:MEK structure (blue) and the previously reported cryo-EM BRAF:14-3-32:MEK1 structure bound to ATP- $\gamma \mathrm{S}$ (PDB ID:6NYB, green). d Superimposition of the BRAF KD:MEK component of the BRAF:14-3$3_{2}$ :MEK in the present structure (BRAF KD in blue and MEK in gray) with the previously reported crystal structure of the isolated BRAF KD:MEK1 dimer (PDB ID: 6U2G, pink), with a $\mathrm{C} \alpha$ R.M.S.D. of $0.97 \AA$. Superimposition of these structures was done based on the BRAF KD:MEK component of the structures. Also shown, is the cryo-EM density corresponding to the MEK inhibitor CH5126766 bound to the allosteric site of MEK. 
a

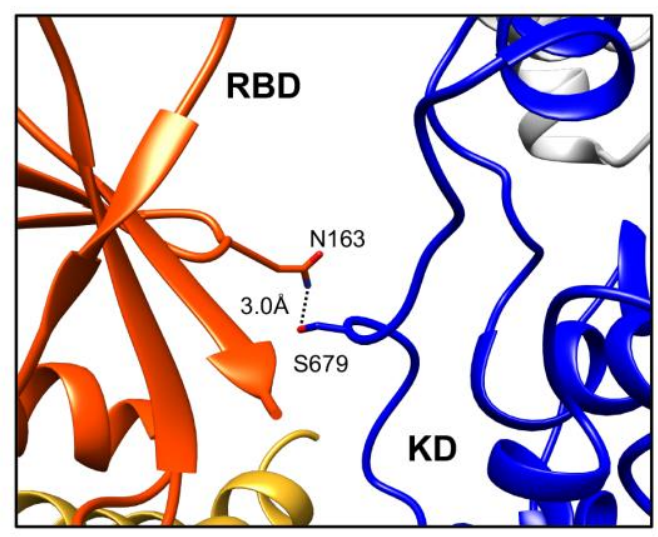

C

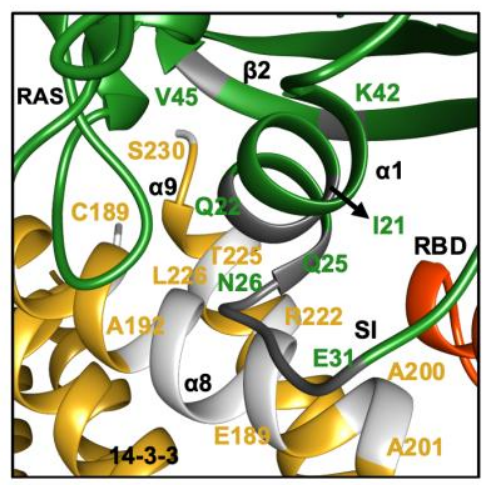

d b
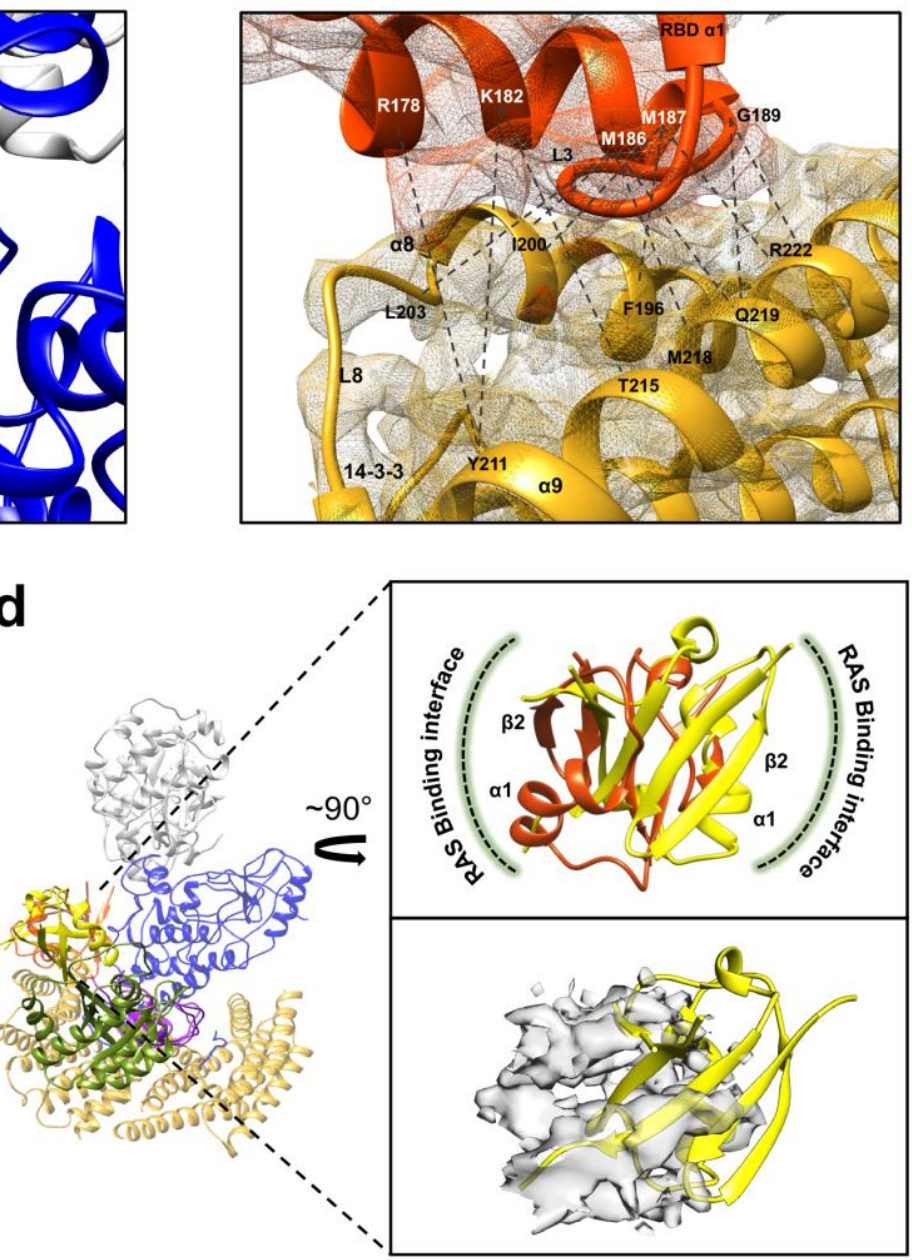

\section{Supplementary Fig. 6 Structural analysis of BRAF RBD interactions}

a Potential interaction between N163 in the BRAF RBD (red orange) and S679 in the BRAF KD (blue). b Density map showing the RBD:14-3-3 interface, with the RBD region colored in orange and the 14-3-3 region colored in gold. The potential interacting residues are labeled $\mathbf{c}$ Potential steric clash at the RBD:14-3-3 interface between RAS residues I21-Q22 ( $\alpha 1$-helix), Q25-E31 (SI region), K42 and V45 ( $\beta 2$ sheet) with 14-3-3 $\alpha 8$-helix residues C189, A192-E198, I200-A201 and a9-helix residues R222, L225-T226 and S230 upon superimposition of KRAS onto the autoinhibited BRAF:14-3-32:MEK complex. RAS is colored in green, 14-3-3 in gold, and the potential clashing residues in RAS and 14-3-3 are colored in dark and light gray respectively. d Rigid body superposition of the BRAF:14-3-32:MEK complex (colored as in Fig. 2) and the 
KRAS:CRAF_RBD-CRD complexes (KRAS in green, CRAFRBD in yellow, and CRAF CRD in violet. PDB ID: 6XI7), with the alignment based on the CRD. Insets show the difference in the RBD orientation (top) and position (bottom) between the two structures. For the RBD position, the cryo-EM density map in the region of the BRAF RBD is shown in light gray and the CRAF $\mathrm{RBD}$ ribbon structure is in yellow. 


\section{Supplementary Tables}

Supplementary Table 1. Fit of BRAF:14-3-32 and BRAF:14-3-32:MEK structures to their corresponding cryo-EM maps showing the inctive conformation of the KD is adopted.

\begin{tabular}{|c|c|c|}
\hline Structural element & $\begin{array}{l}\text { Structural fit of BRAF:14-3-32 } \\
\text { (PDB ID 7MFE) to } \\
\text { corresponding cryo-EM density } \\
\text { map (EMD-23814) }\end{array}$ & $\begin{array}{c}\text { Structure fit of BRAF:14-3- } \\
\text { 32:MEK (PDB ID 7MFD) to } \text { :ME } \\
\text { corresponding cryo-EM density } \\
\text { (EMD-23813) }\end{array}$ \\
\hline \multicolumn{3}{|l|}{$\begin{array}{l}\text { Representative } \\
\text { regions in the KD C- } \\
\text { lobe }\end{array}$} \\
\hline \multicolumn{3}{|l|}{$\begin{array}{l}\alpha \text { C- helix in "out" } \\
\text { conformation } \\
\text { The distance between the "in" } \\
\text { and "out" position for the } \alpha \mathrm{C}- \\
\text { helix is } \sim 6-7 \AA \text {. The } \alpha \mathrm{C}- \\
\text { helix fits well in the "out" } \\
\text { position, as is observed in } \\
\text { PDBs 6NYB (Park } \text { et al., } \\
\text { 2019) and 6U2G (Liu } \text { et al., } \\
\text { 2020). }\end{array}$} \\
\hline \multirow{2}{*}{\multicolumn{3}{|c|}{$\begin{array}{l}\text { Inhibitory turn } \\
\text { (Residues A598-S602) } \\
\text { Similar to PDB 6NYB (Park } \\
\text { et al., 2019), the inhibitory } \\
\text { turn is observed. }\end{array}$}} \\
\hline & & \\
\hline \multirow{3}{*}{$\begin{array}{c}\text { Broken R spine } \\
\text { (with L505 misaligned) } \\
\text { Similar to PDBs 6NYB (Park } \\
\text { et al., 2019) and 6U2G (Liu et } \\
\text { al., 2020), the R-spine is } \\
\text { broken. }\end{array}$} & $L^{505}$ & \\
\hline & $\mathrm{F}^{595}$ & $\mathrm{~F}^{595}$ \\
\hline & (Fit of L505 is shown above) & (Fit of L505 is shown above) \\
\hline
\end{tabular}


bioRxiv preprint doi: https://doi.org/10.1101/2021.09.30.462573; this version posted December 30, 2021. The copyright holder for this preprint (which was not certified by peer review) is the author/funder. All rights reserved. No reuse allowed without permission.

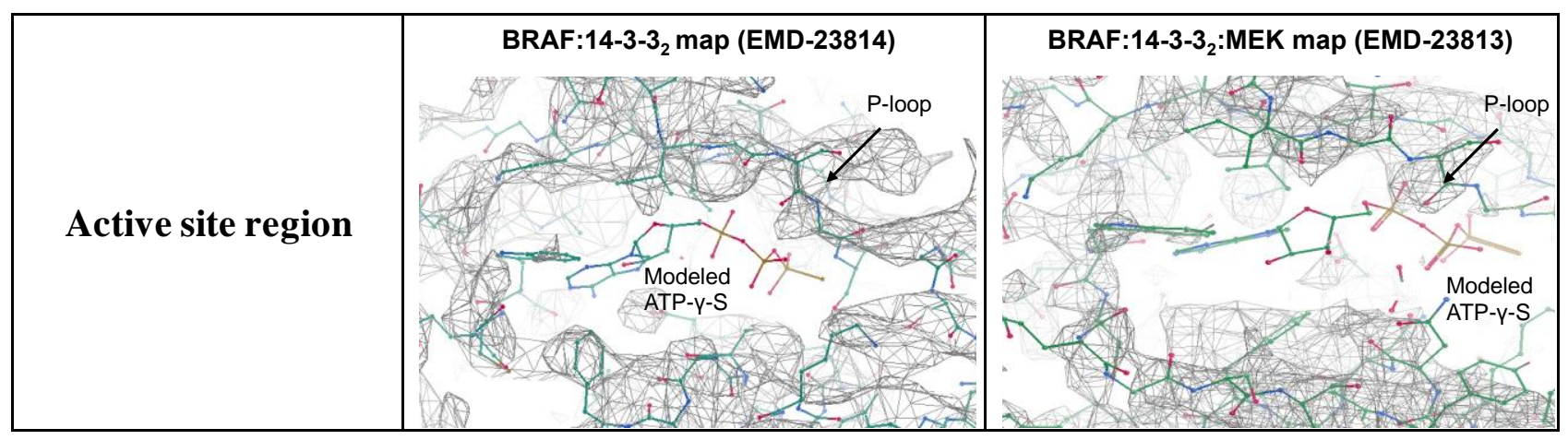


Supplementary Table 2. Fitting of active and inactive BRAF KD conformations onto the KD density map of BRAF:14-3-3 and BRAF:14-3-32:MEK

BRAF KD active (PBD ID 2FB8) (left) and inactive (PDB ID 6U2G) (middle) structures fitted onto the BRAF:14-3-32 (EMD-23814) KD density map and compared with that in the BRAF:14-3-32 (PDB ID 7MFE) structure in this study (right)

Regions with unoccupied or misfitted densities are indicated by dashed lines. Two views with different orientations a re shown.

View 1

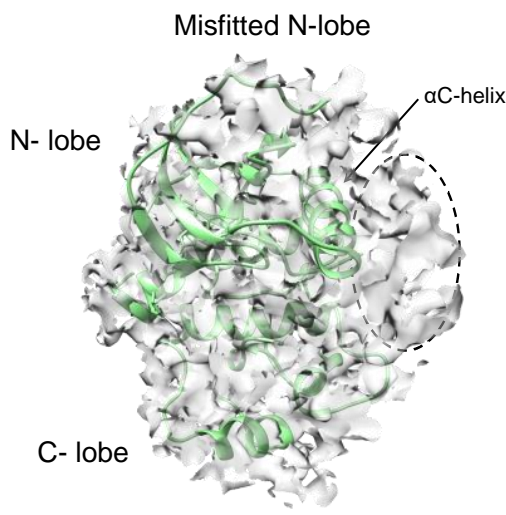

Active BRAF KD (2FB8)

EMD-23814 map (BRAF:14-3-32)

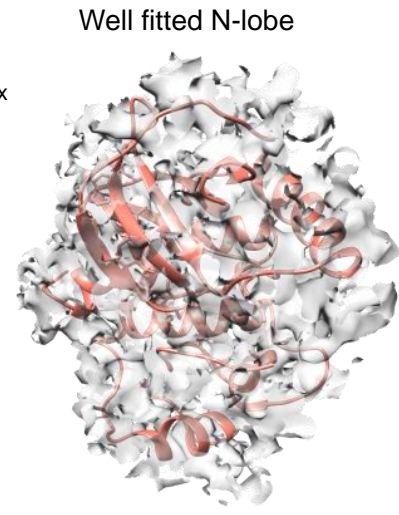

Inactive BRAF KD (6U2G)

EMD-23814 map (BRAF:14-3-32)

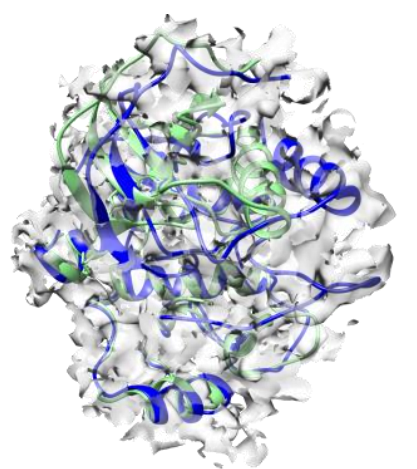

Active BRAF KD (2FB8) vs KD on BRAF:(14-3-3) 2 (7MFE)

EMD-23814 map (BRAF:14-3-32)

View 2

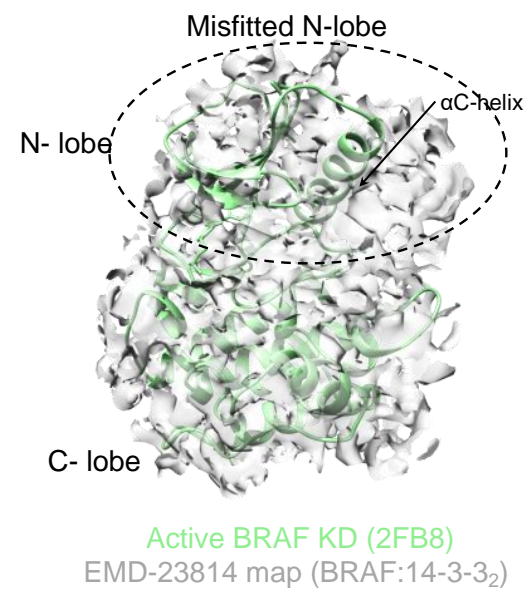

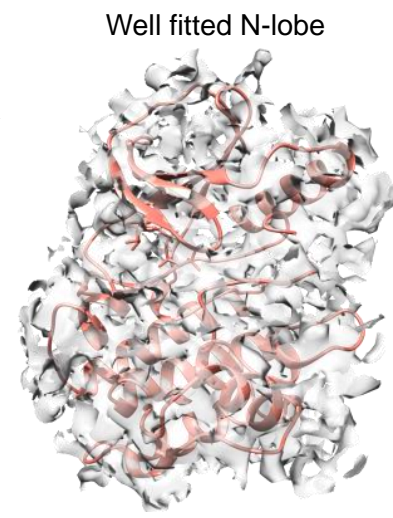

Inactive BRAF KD (6U2G) EMD-23814 map (BRAF:14-3-32)

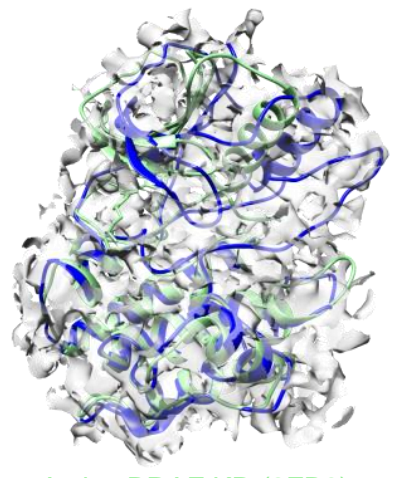

Active BRAF KD (2FB8) vs KD on BRAF:14-3-3 2 (7MFE) 
BRAF KD active (PBD ID 2FB8) (left) and inactive (PDB ID 6U2G) (middle) conformations fitted onto the BRAF:14-3-32:MEK (EMD-23813) map at the KD region and compared with that in the BRAF:14-3-32:MEK (PDB ID 7MFD) structure in this study (right)

Regions with unoccupied or misfitted densities a re indicated by dashed lines. Two views with different orientations are shown.
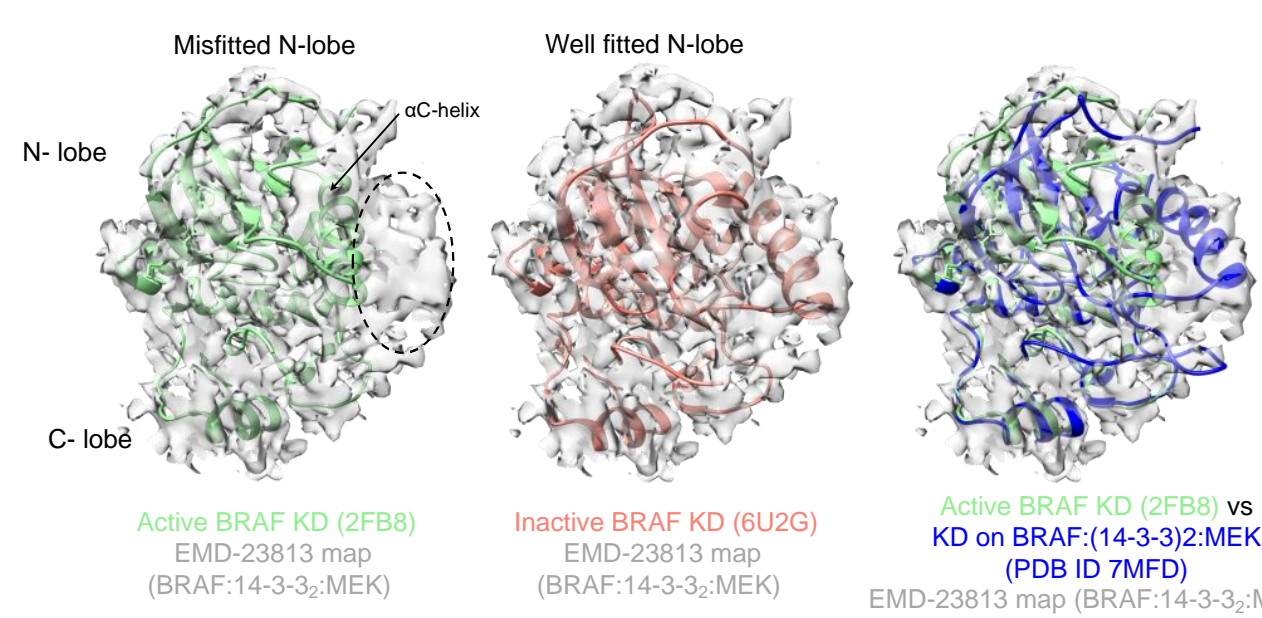

Active BRAF KD (2FB8) vs

KD on BRAF:(14-3-3)2:MEK (PDB ID 7MFD)

EMD-23813 map (BRAF:14-3-32:MEK)

View 2
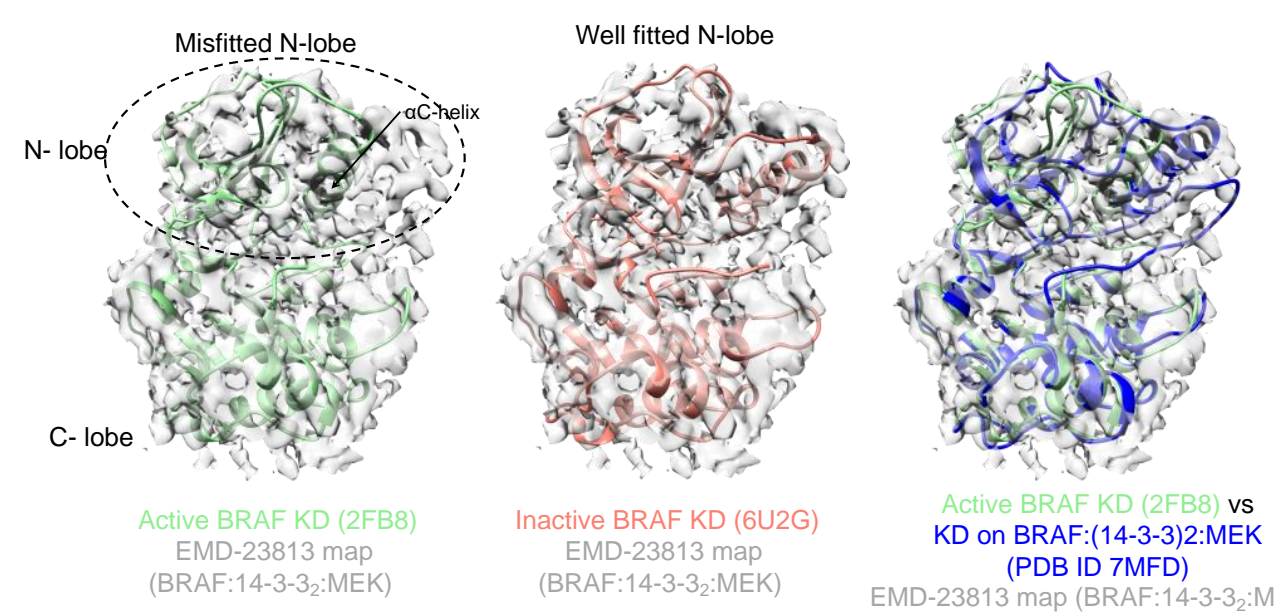

EMD-23813 map (BRAF:14-3-32:MEK)

\section{Orientation of the $\mathrm{N}$-lobe relative to the $\mathrm{C}$-lobe}

Conformation is similar to that in PDBs 6NYB (Park et al., 2019) and 6U2G (Liu et al., 2020) with the N- and C- lobes exhibiting $\sim 13^{\circ}$ closer orientation than is observed in RAF-inhibitor-bound KDs (4RZV) 
bioRxiv preprint doi: https://doi.org/10.1101/2021.09.30.462573; this version posted December 30, 2021. The copyright holder for this preprint (which was not certified by peer review) is the author/funder. All rights reserved. No reuse allowed without permission.

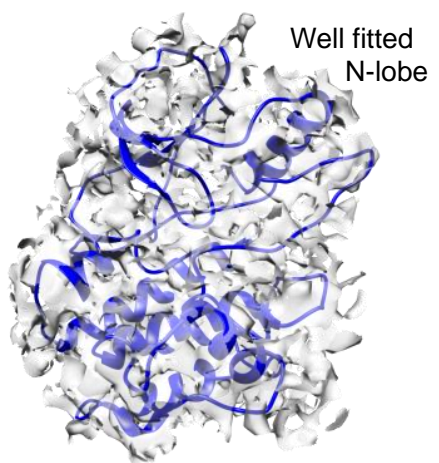

KD on BRAF:14-3-3 2 (7MFE)

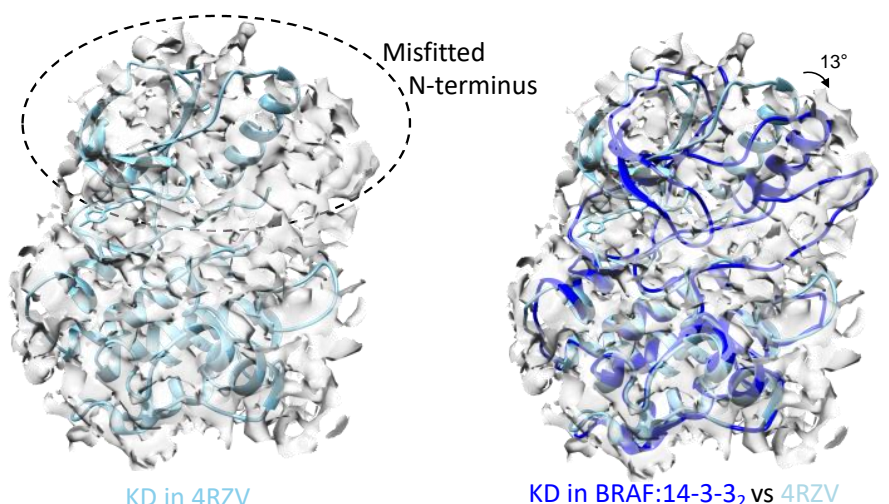

KD in BRAF:14-3-3, vs $4 R Z V$ 
Supplementary Table 3. Reagent or Resource Table

\begin{tabular}{|c|c|c|}
\hline REAGENT or RESOURCE & SOURCE & IDENTIFIER \\
\hline \multicolumn{3}{|l|}{ Antibodies } \\
\hline HaloTag mouse monoclonal & Promega & cat\# G9211; RRID:AB_2688011 \\
\hline Rluc rabbit polyclonal & MBL International & cat\# PM047; RRID:AB_1520866 \\
\hline GFP mouse monoclonal & Roche & $\begin{array}{ll}\text { cat\# } & 11814460001 ; \\
\text { RRID:AB_390913 } & \\
\end{array}$ \\
\hline GFP rat monoclonal & MBL International & cat\# D153-3; RRID:AB_591817 \\
\hline RAS10 mouse monoclonal & Millipore & cat\# 05-516; RRID:AB_11211664 \\
\hline BRAF F-7 mouse monoclonal & $\begin{array}{l}\text { Santa Cruz } \\
\text { Biotechnology }\end{array}$ & cat\# sc-5284; RRID:AB_626760 \\
\hline $\begin{array}{l}\text { pS217/221-MEK rabbit } \\
\text { polyclonal }\end{array}$ & $\begin{array}{l}\text { Cell Signaling } \\
\text { Technology }\end{array}$ & cat\# 9121; RRID: AB_331648 \\
\hline MEK 1 mouse monoclonal & BD Biosciences & cat\# 610122; RRID:AB_397528 \\
\hline MEK 2 mouse monoclonal & BD Biosciences & cat\# 610236; RRID:AB_397631 \\
\hline \multicolumn{3}{|l|}{ Critical Commercial Assays } \\
\hline Superose $6,10 / 300 \mathrm{GL}$ & Cytiva & cat\# 29091596 \\
\hline Pierce Silver Stain Kit & Thermo Scientific & cat\# 24612 \\
\hline NanoBRET Kit & Promega & cat\# N1661 \\
\hline Halolink Resin & Promega & cat\# G1915 \\
\hline Halo-TEV & Promega & cat\# G6602 \\
\hline \multicolumn{3}{|l|}{ Deposited Data } \\
\hline BRAF:14-3-32:MEK & This paper & $\begin{array}{l}\text { Coordinates: PDBID: 7MFD } \\
\text { Cryo-EM map: EMDB: EMD- } \\
23813\end{array}$ \\
\hline BRAF:14-3-3 & This paper & $\begin{array}{l}\text { Coordinates: PDBID: 7MFE } \\
\text { Cryo-EM map: EMDB: EMD- } \\
23814\end{array}$ \\
\hline $\mathrm{BRAF}_{2}: 14-3-3_{2}$ & This paper & $\begin{array}{l}\text { Coordinates: PDBID: 7MFF } \\
\text { Cryo-EM map: EMDB: EMD- } \\
23815\end{array}$ \\
\hline $\begin{array}{l}\text { Coordinates of RAF-1 cysteine- } \\
\text { rich domain }\end{array}$ & $(\text { Moot et al., 1996) })^{41}$ & PDB ID: 1FAR \\
\hline $\begin{array}{l}\text { Coordinates of BRAF kinase } \\
\text { domain bound to SB-590885 }\end{array}$ & (King et al., 2006) $^{43}$ & PDB ID: 2FB8 \\
\hline $\begin{array}{l}\text { Coordinates of Human MEK1 } \\
\text { kinase in complex with } \\
\text { CH5126766 and MgAMP-PNP }\end{array}$ & $(\text { Lito et al., 2014) })^{42}$ & PDB ID: $3 \mathrm{WIG}$ \\
\hline $\begin{array}{l}\text { Coordinates of } 14-3-3 \text { isoform } \\
\text { zeta in complex with a }\end{array}$ & $\begin{array}{l}\text { (Molzan and Ottmann, } \\
2012)^{44}\end{array}$ & PDB ID: 4FJ3 \\
\hline
\end{tabular}




\begin{tabular}{|c|c|c|}
\hline $\begin{array}{l}\text { diphoyphorylated CRAF } \\
\text { peptide }\end{array}$ & & \\
\hline $\begin{array}{l}\text { Coordinates of the } \\
\text { BRAF:MEK1 complex }\end{array}$ & (Haling et al., 2016) 34 & PDB ID: 4MNE \\
\hline $\begin{array}{l}\text { Coordinates of BRAF }(\mathrm{R} 509 \mathrm{H}) \\
\text { kinase domain monomer bound } \\
\text { to Vemurafenib }\end{array}$ & $\left(\right.$ Karoulia et al., 2016) ${ }^{27}$ & PDB ID: 4RZV \\
\hline $\begin{array}{l}\text { Coordinates of RAS Binding } \\
\text { Domain (RBD) of BRAF }\end{array}$ & $\begin{array}{l}\text { (Athuluri-Divakar et al., } \\
2016)^{40}\end{array}$ & PDB ID: 5J17 \\
\hline $\begin{array}{l}\text { Coordinates of BRAF:MEK1: } \\
14-3-3\end{array}$ & (Park et al., 2019) $^{17}$ & PDB ID: 6NYB \\
\hline $\begin{array}{l}\text { Coordinates of BRAF:14-3-3 } \\
\text { dimer complex }\end{array}$ & (Kondo et al., 2019) $^{18}$ & PDB ID: 6UAN \\
\hline $\begin{array}{l}\text { Coordinates of BRAF:MEK } \\
\text { complex with AMP-PCP }\end{array}$ & $(\text { Liau et al., 2020) })^{20}$ & PDB ID: $6 \mathrm{U} 2 \mathrm{G}$ \\
\hline $\begin{array}{l}\text { Coordinates of BRAF dimer } \\
\text { bound to } 14-3-3\end{array}$ & $(\text { Liau et al., 2020) })^{20}$ & PDB ID: $6 \mathrm{U} 2 \mathrm{H}$ \\
\hline $\begin{array}{l}\text { Coordinates of } \\
\text { KRAS:CRAF_RBD-CRD } \\
\text { complex }\end{array}$ & $($ Tran et al., 2021) 32 & PDB ID: 6XI7 \\
\hline Coordinates of RAS:RBD-CRD & $\begin{array}{l}\text { (Cookis and Mattos, } \\
2021)^{35}\end{array}$ & PDB ID: 7JHP \\
\hline \multicolumn{3}{|l|}{ Experimental Models: Cell lines } \\
\hline 293FT (human) & ATCC & cat\# PTA-5077, RRID:CVCL_6911 \\
\hline 293T (human) & ATCC & $\begin{array}{ll}\text { cat\# } & \text { CRL-11268; } \\
\text { RRID:CVCL_1926 } & \end{array}$ \\
\hline HeLa (human, female) & ATCC & cat\# CCL-2; RRID:CVCL_0030 \\
\hline Phoenix-Eco (human) & ATCC & $\begin{array}{ll}\text { cat\# } & \text { CRL-3214; } \\
\text { RRID:CVCL_H717 } & \\
\end{array}$ \\
\hline NIH-3T3 (mouse) & ATCC & $\begin{array}{ll}\text { cat\# } & \text { CRL-1658; } \\
\text { RRID:CVCL_0594 } & \\
\end{array}$ \\
\hline \multicolumn{3}{|l|}{ Recombinant DNA } \\
\hline pCMV5-Halo-BRAFWT & NCI-Ras Initiative & N/A \\
\hline pCMV5-Venus-KRAS ${ }^{\mathrm{G} 12 \mathrm{~V}}$ & NCI-Ras Initiative & N/A \\
\hline pCMV5-Halo-KRASG12V & NCI-Ras Initiative & N/A \\
\hline $\begin{array}{l}\text { Lenti-puro-CMV-HA- } \\
\text { KRASG12V }\end{array}$ & NCI-Ras Initiative & N/A \\
\hline pLHCX-BRAFFL-RLuc8 & $($ Terrell et al., 2019)33 & N/A \\
\hline $\begin{array}{l}\text { pLHCX-BRAFFL R158A- } \\
\text { RLuc8 }\end{array}$ & This paper & N/A \\
\hline $\begin{array}{l}\text { pLHCX-BRAFFL R166A- } \\
\text { RLuc8 }\end{array}$ & This paper & N/A \\
\hline
\end{tabular}




\begin{tabular}{|c|c|c|}
\hline $\begin{array}{l}\text { pLHCX-BRAFFL K183A- } \\
\text { RLuc8 }\end{array}$ & This paper & N/A \\
\hline $\begin{array}{l}\text { pLHCX-BRAFFL R188L- } \\
\text { RLuc8 }\end{array}$ & This paper & N/A \\
\hline $\begin{array}{l}\text { pLHCX-BRAFFL } \\
\text { M186A/M187A-RLuc8 }\end{array}$ & This paper & N/A \\
\hline $\begin{array}{l}\text { pLHCX-BRAFFL } \\
\text { M186W/M187W-RLuc8 }\end{array}$ & This paper & N/A \\
\hline $\begin{array}{l}\text { pLHCX-BRAFFL } \\
\text { M186K/M187V-RLuc8 }\end{array}$ & This paper & N/A \\
\hline pCMV5-BRAFREG_Halo & This paper & $\mathrm{N} / \mathrm{A}$ \\
\hline $\begin{array}{l}\text { pCMV5- BRAFREG } \\
\text { M186A/M187A -Halo }\end{array}$ & This paper & N/A \\
\hline $\begin{array}{l}\text { pCMV5- BRAFREG } \\
\text { M186W/M187W -Halo }\end{array}$ & This paper & N/A \\
\hline $\begin{array}{l}\text { pCMV5- BRAFREG } \\
\text { M186K/M187V -Halo }\end{array}$ & This paper & N/A \\
\hline $\begin{array}{l}\text { pCMV5- BRAFREG T241P - } \\
\text { Halo }\end{array}$ & This paper & N/A \\
\hline pCMV5-NanoLuc-CRAFCat & This paper & $\mathrm{N} / \mathrm{A}$ \\
\hline pCMV5-BRAFRBD_Halo & This paper & N/A \\
\hline $\begin{array}{l}\text { pCMV5- BRAFRBD } \\
\text { M186A/M187A -Halo }\end{array}$ & This paper & N/A \\
\hline $\begin{array}{l}\text { pCMV5- BRAFRBD } \\
\text { M186W/M187W -Halo }\end{array}$ & This paper & N/A \\
\hline $\begin{array}{l}\text { pCMV5- BRAFRBD } \\
\text { M186K/M187V -Halo }\end{array}$ & This paper & N/A \\
\hline pBABE-puro-Flag-BRAF & $\left(\right.$ Terrell et al., 2019) ${ }^{33}$ & $\mathrm{N} / \mathrm{A}$ \\
\hline $\begin{array}{l}\text { pBABE-puro-Flag-BRAF } \\
\text { M186A/M187A }\end{array}$ & This paper & N/A \\
\hline $\begin{array}{l}\text { pBABE-puro-Flag-BRAF } \\
\text { M186W/M187W }\end{array}$ & This paper & N/A \\
\hline $\begin{array}{l}\text { pBABE-puro-Flag-BRAF } \\
\text { T241P }\end{array}$ & This paper & N/A \\
\hline \multicolumn{3}{|l|}{ Software and Algorithms } \\
\hline Serial EM 3-7-6-64 & (Mastronarde, 2005) ${ }^{36}$ & http://bio3d.colorado.edu/SerialEM \\
\hline Relion 3.1 & (Zivanov et al., 2018) ${ }^{37}$ & $\begin{array}{l}\text { https://www3.mrc- } \\
\text { lmb.cam.ac.uk/relion/index.php/Ma } \\
\text { in_Page }\end{array}$ \\
\hline MotionCor2 & (Zheng et al., 2017) ${ }^{38}$ & $\begin{array}{l}\text { http://msg.ucsf.edu/em/software/m } \\
\text { otioncor2.html }\end{array}$ \\
\hline Gctf & (Zhang, 2016) ${ }^{39}$ & $\begin{array}{l}\text { https://www2.mrc- } \\
\text { lmb.cam.ac.uk/research/locally- } \\
\text { developed-software/zhang- } \\
\text { software/ }\end{array}$ \\
\hline
\end{tabular}




\begin{tabular}{|c|c|c|}
\hline COOT 0.9 .6 & $\begin{array}{l}\text { (Emsley and Cowtan, } \\
2004) 46\end{array}$ & $\begin{array}{l}\text { https://www2.mrc- } \\
\text { lmb.cam.ac.uk/personal/pemsley/co } \\
\text { ot }\end{array}$ \\
\hline PHENIX 1.17.1 & (Adams et al., 2010) ${ }^{47}$ & https://www.phenix-online.org \\
\hline Chimera 1.13.1 & (Pettersen et al., 2004) ${ }^{45}$ & https://www.cgl.ucsf.edu/chimera \\
\hline Prism 8 & N/A & $\begin{array}{l}\text { https://www.graphpad.com/scientif } \\
\text { ic-software/prism }\end{array}$ \\
\hline \multicolumn{3}{|l|}{ Other } \\
\hline $\begin{array}{l}\mathrm{R} 1.2 / 1.3300 \text { mesh Au holey } \\
\text { carbon grids }\end{array}$ & $\begin{array}{l}\text { Electron Microscopy } \\
\text { Sciences }\end{array}$ & Cat\# Q350AR1.3 \\
\hline
\end{tabular}


Supplementary Table 4. Cryo-EM data collection, Refinement and Validation Statistics

\begin{tabular}{|c|c|c|c|}
\hline & BRAF:14-3-32:MEK & BRAF:14-3-32 & BRAF $2: 14-3-32$ \\
\hline \multicolumn{4}{|l|}{ Data Processing } \\
\hline Data availability & $\begin{array}{l}\text { PDB ID: 7MFD, } \\
\text { EMB-23813 }\end{array}$ & $\begin{array}{c}\text { PDB ID: 7MFE, } \\
\text { EMB-23814 }\end{array}$ & $\begin{array}{l}\text { PDB ID: 7MFF, } \\
\text { EMB-23815 }\end{array}$ \\
\hline Magnification & 130k, EFTEM mode & 105k, EFTEM mode & 105k, EFTEM mode \\
\hline Voltage (kV) & 300 & 300 & 300 \\
\hline Electron exposure $\left(\mathrm{e}^{-} / \AA^{2}\right)$ & 57 & 55 & 55 \\
\hline Exposure Rate $\left(\mathrm{e}^{-} / \AA^{2} / \mathrm{s}\right)$ & 7.13 & 5.5 & 5.5 \\
\hline Dose/frame $\left(\mathrm{e}^{-} / \AA^{2}\right)$ & 1.14 & 1.10 & 1.10 \\
\hline \multicolumn{4}{|l|}{ Collection } \\
\hline $\begin{array}{l}\text { Number of frames } \\
\text { collected per micrograph }\end{array}$ & 50 & 50 & 50 \\
\hline Energy filter slit width & $20 \mathrm{eV}$ & $20 \mathrm{eV}$ & $20 \mathrm{eV}$ \\
\hline Defocus range $(\mu \mathrm{m})$ & -0.8 to -2.5 & -0.8 to -2.5 & -0.8 to -2.5 \\
\hline Pixel size $(\AA)$ & 1.058 & 1.348 & 1.348 \\
\hline Symmetry imposed & $\mathrm{C} 1$ & $\mathrm{C} 1$ & C1 \\
\hline Movies (no.) & 3,976 & 5995 & 2,998 \\
\hline $\begin{array}{l}\text { Initial particle images } \\
\text { (no.) }\end{array}$ & $1,824,538$ & $2,496,302$ & $2,159,824$ \\
\hline $\begin{array}{l}\text { Final particle images } \\
\text { (no.) }\end{array}$ & 142,852 & 198,731 & 203,343 \\
\hline Map resolution $(\AA)$ & 3.66 & 4.07 & 3.89 \\
\hline FSC threshold & & 0.143 & \\
\hline \multicolumn{4}{|l|}{ Refinement } \\
\hline $\begin{array}{l}\text { Initial model used (PDB } \\
\text { ID) }\end{array}$ & $\begin{array}{c}\text { 1FAR, 3WIG, 4FJ3, } \\
\text { 4MNE, 5J17 }\end{array}$ & $\begin{array}{c}\text { 1FAR, 4FJ3, 4MNE, } \\
5 \mathrm{~J} 17\end{array}$ & 2FB8, 4FJ3 \\
\hline $\begin{array}{l}\text { Map sharpening B factor } \\
\left(\AA^{2}\right)\end{array}$ & -100.5 & -168.6 & -167.4 \\
\hline \multicolumn{4}{|l|}{ Model composition } \\
\hline Non-hydrogen atoms & 9127 & 6843 & 8008 \\
\hline Protein residues & 1147 & 859 & 1000 \\
\hline Ligands & 1 & 0 & 2 \\
\hline Metals & 2 & 2 & 0 \\
\hline \multicolumn{4}{|l|}{ B factors $\left(\AA^{2}\right)$} \\
\hline Protein & 80.3 & 35.9 & 41.8 \\
\hline Ligand & 94.6 & 42.2 & 23.6 \\
\hline \multicolumn{4}{|l|}{ r.m.s deviations } \\
\hline Bond lengths $(\AA)$ & 0.004 & 0.002 & 0.002 \\
\hline Bond angles $\left({ }^{\circ}\right)$ & 0.659 & 0.570 & 0.522 \\
\hline \multicolumn{4}{|l|}{ Validation statistics } \\
\hline MolProbity score & 2.19 & 2.18 & 1.69 \\
\hline
\end{tabular}




\begin{tabular}{|l|c|c|c|}
\hline Clash Score & 17.6 & 17.8 & 8.6 \\
\hline Rotamer outliers (\%) & 0 & 0 & 0.12 \\
\hline Ramachandran Plot & & & 96.5 \\
\hline Favored (\%) & 93.0 & 93.4 & 3.5 \\
\hline Allowed (\%) & 7.0 & 0.6 & 0 \\
\hline Outliers (\%) & 0 & & 0.71 \\
\hline Model vs Data & & 0.64 & 0.69 \\
\hline CC (mask) & 0.61 & 0.63 & 0.61 \\
\hline CC (box) & 0.68 & 0.50 & 0.69 \\
\hline CC (peaks) & 0.53 & 0.62 & 0.71 \\
\hline CC (volume) & 0.60 & 0.72 & \\
\hline Mean CC for ligands & 0.69 & & \\
\hline
\end{tabular}

\section{Supplementary References}

1 Cox, A. D. \& Der, C. J. Ras history: The saga continues. Small GTPases 1, 2-27, doi:10.4161/sgtp.1.1.12178(2010).

2 Lavoie, H. \& Therrien, M. Regulation of RAF protein kinases in ERK signalling. Nature reviews. Molecular cell biology 16,281-298, doi:10.1038/nrm3979(2015).

3 Terrell, E. M. \& Morrison, D. K. Ras-Mediated Activation of the Raf Family Kinases. Cold Spring Harbor perspectives in medicine 9, doi:10.1101/cshperspect.a033746 (2019).

4 Nan, X. et al. Single-molecule superresolution imaging allows quantitative analysis of RAF multimer formation and signaling. Proceedings of the National Academy of Sciences of the United States of America 110, 18519-18524, doi:10.1073/pnas.13181881 10 (2013).

5 Cutler, R. E. J., Stephens, R. M., Saracino, M. R. \& Morrison, D. K. Autoregulation of the Raf-1 serine/threonine kinase. Proc Natl Acad Sci USA 95, 9214-9219 (1998).

6 Guan, K.-L. et al. Negative regulation of the serine/threonine kinase B-Raf by Akt. J. Biol. Chem. 275, 27354-27359 (2000).

7 Tran, N. H., Wu, X. \& Frost, J. A. B-Raf and Raf-1 are regulated by distinct autoregulatory mechanisms. J Biol Chem 280, 16244-16253, doi:10.1074/jbc.M501185200 (2005).

8 Freeman, A. K. \& Morrison, D. K. 14-3-3 Proteins: diverse functions in cell proliferation and cancer progression. Semin Cell Dev Biol 22, 681-687, doi:10.1016/j.semcdb.2011.08.009 (2011).

9 Van Aelst, L., Barr, M., Marcus, S., Polverino, A. \& Wigler, M. Complex formation between Ras and Raf and other protein kinases. Proc. Natl. Acad. Sci. USA 90, 6213-6217 (1993).

10 Vojtek, A. B., Hollenberg, S. M. \& Cooper, J. A. Mammalian Ras interacts directly with the serine/threonine kinase Raf. Cell 74, 205-214 (1993).

11 Zhang, X. F. et al. Normal and oncogenic p21 ras proteins bind to the amino-terminal regulatory domain of c-Raf-1. Nature 364,308-313, doi:10.1038/364308a0 (1993).

12 Marais, R., Light, Y., Paterson, H. F. \& Marshall, C. J. Ras recruits Raf-1 to the plasma membrane for activation by tyrosine phosphorylation. Embo j 14, 3136-3145 (1995).

13 Weber, C. K., Slupsky, J. R., Kalmes, H. A. \& Rapp, U. R. Active Ras induces heterodimerization of cRaf and BRaf. Cancer Res 61, 3595-3598 (2001). 
14 Rushworth, L. K., Hindley, A. D., O'Neill, E. \& Kolch, W. Regulation and role of Raf -1/BRaf heterodimerization. Mol Cell Biol 26, 2262-2272, doi:10.1128/mcb.26.6.22622272.2006 (2006).

15 Freeman, A. K., Ritt, D. A. \& Morrison, D. K. Effects of Raf dimerization andits inhibition on normal and disease-associated Raf signaling. Mol Cell 49, 751-758, doi:10.1016/j.molcel.2012.12.018 (2013).

$16 \mathrm{Hu}, \mathrm{J}$. et al. Allosteric activation of functionally asymmetric RAF kinase dimers. Cell 154, 1036-1046, doi:10.1016/j.cell.2013.07.046(2013).

17 Park, E. et al. Architecture of autoinhibited and active BRAF-MEK1-14-3-3 complexes. Nature, doi:10.1038/s41586-019-1660-y (2019).

18 Kondo, Y. et al. Cryo-EM structure of a dimeric B-Raf:14-3-3 complex reveals asymmetry in the active sites of B-Raf kinases. Science 366, 109-115, doi:10.1126/science.aay0543 (2019).

19 Liau, N. P. D. et al. Dimerization Induced by C-Terminal 14-3-3 Binding Is Sufficient for BRAF Kinase Activation. Biochemistry 59,3982-3992, doi:10.1021/acs.biochem.0c00517 (2020).

20 Liau, N. P. D. et al. Negative regulation of RAF kinase activity by ATP is overcome by 14-3-3-induced dimerization. Nat Struct Mol Biol 27, 134-141, doi:10.1038/s41594-0190365-0 (2020).

21 Geiger, T., Wehner, A., Schaab, C., Cox, J. \& Mann, M. Comparative proteomic analysis of eleven common cell lines reveals ubiquitous but varying expression of most proteins. Mol Cell Proteomics 11, M111.014050, doi:10.1074/mcp.M111.014050 (2012).

22 Yang, X. et al. Structural basis for protein-protein interactions in the 14-3-3 protein family. Proceedings of the National Academy of Sciences of the United States of America 103, 17237-17242, doi:10.1073/pnas.0605779103 (2006).

23 Rajakulendran, T., Sahmi, M., Lefrancois, M., Sicheri, F. \& Therrien, M. A dimerizationdependent mechanism drives RAF catalytic activation. Nature 461, 542-545, doi:10.1038/nature08314 (2009).

24 McClendon, C. L., Kornev, A. P., Gilson, M. K. \& Taylor, S. S. Dynamic architecture of a protein kinase. Proceedings of the National Academy of Sciences of the United States of America 111, E4623-4631, doi:10.1073/pnas.1418402111 (2014).

25 Thevakumaran, N. et al. Crystal structure of a BRAF kinase domain monomer explains basis for allosteric regulation. Nat Struct Mol Biol 22, 37-43, doi:10.1038/nsmb.2924 (2015).

26 Peng, S. B. et al. Inhibition of RAF Isoforms and Active Dimers by LY3009120 Leads to Anti-tumor Activities in RAS or BRAF Mutant Cancers. Cancer Cell 28, 384-398, doi:10.1016/j.ccell.2015.08.002(2015).

27 Karoulia, Z. et al. An Integrated Model of RAF Inhibitor Action Predicts Inhibitor Activity against Oncogenic BRAF Signaling. Cancer Cell 30, 485-498, doi:10.1016/j.ccell.2016.06.024 (2016).

28 Fischmann, T. O. et al. Crystal structures of MEK1 binary and ternary complexes with nucleotides and inhibitors. Biochemistry 48, 2661-2674, doi:10.1021/bi801898e (2009).

29 Nassar, N. et al. The 2.2 A crystal structure of the Ras-binding domain of the serine/threonine kinase c-Raf1 in complex with Rap1A and a GTP analogue. Nature 375, 554-560, doi:10.1038/375554a0 (1995). 
30 Herrmann, C., Martin, G. A. \& Wittinghofer, A. Quantitative analysis of the complex between p21ras and the Ras-binding domain of the human Raf-1 protein kinase. $J$ Biol Chem 270, 2901-2905. (1995).

31 Fetics, S. K. et al. Allosteric effects of the oncogenic RasQ61L mutant on Raf-RBD. Structure 23, 505-516, doi:10.1016/j.str.2014.12.017 (2015).

32 Tran, T. H. et al. KRAS interaction with RAF1 RAS-binding domain and cysteine-rich domain provides insights into RAS-mediated RAF activation. Nat Commun 12, 1176, doi:10.1038/s41467-021-21422-x (2021).

33 Terrell, E. M. et al. Distinct Binding Preferences between Ras and Raf Family Members and the Impact on Oncogenic Ras Signaling. Mol Cell 76, 872-884 e875, doi:10.1016/j.molcel.2019.09.004 (2019).

34 Haling, J. R. et al. Structure of the BRAF-MEK complex reveals a kinase activity independent role for BRAF in MAPK signaling. Cancer Cell 26, 402-413, doi:10.1016/j.ccr.2014.07.007 (2014).

35 Cookis, T. \& Mattos, C. Crystal structure reveals the full Ras-Raf interface and advances mechanistic understanding of Raf activation. Biomolecules 11, 996, doi:10.3390/biom11070996(2021).

36 Mastronarde, D. N. Automated electron microscope tomography using robust prediction of specimen movements. J Struct Biol 152, 36-51, doi:10.1016/j.jsb.2005.07.007 (2005).

37 Zivanov, J. et al. New tools for automated high-resolution cryo-EM structure determination in RELION-3. Elife 7, doi:10.7554/eLife.42166 (2018).

38 Zheng, S. Q. et al. MotionCor2: anisotropic correction of beam-induced motion for improved cryo-electron microscopy. Nat Methods 14, 331-332, doi:10.1038/nmeth.4193 (2017).

39 Zhang, K. Gctf: Real-time CTF determination and correction. J Struct Biol 193, 1-12, doi:10.1016/j.jsb.2015.11.003 (2016).

40 Athuluri-Divakar, S. K. et al. A Small Molecule RAS-Mimetic Disrupts RAS Association with Effector Proteins to Block Signaling. Cell 165, 643-655, doi:10.1016/j.cell.2016.03.045 (2016).

41 Mott, H. R. et al. The solution structure of the Raf-1 cysteine-rich domain: a novel ras and phospholipid binding site. Proceedings of the National Academy of Sciences of the United States of America 93, 8312-8317 (1996).

42 Lito, P. et al. Disruption of CRAF-mediated MEK activation is required for effective MEK inhibition in KRAS mutant tumors. Cancer Cell 25, 697-710, doi:10.1016/j.ccr.2014.03.011(2014).

43 King, A. J. et al. Demonstration of a genetic therapeutic index for tumors expressing oncogenic BRAF by the kinase inhibitor SB-590885. Cancer Res 66, 11100-11105, doi:10.1158/0008-5472.Can-06-2554 (2006).

44 Molzan, M. \& Ottmann, C. Synergistic binding of the phosphorylated S233 - and S259binding sites of C-RAF to one 14-3-3 $\zeta$ dimer. $J$ Mol Biol 423, 486-495, doi:10.1016/j.jmb.2012.08.009(2012).

45 Pettersen, E. F. et al. UCSF Chimera--a visualization system for exploratory research and analysis. J Comput Chem 25, 1605-1612, doi:10.1002/jcc.20084 (2004).

46 Emsley, P. \& Cowtan, K. Coot: model-building tools for molecular graphics. Acta Crystallogr D Biol Crystallogr 60, 2126-2132, doi:10.1107/s0907444904019158 (2004). 
bioRxiv preprint doi: https://doi.org/10.1101/2021.09.30.462573; this version posted December 30, 2021. The copyright holder for this preprint (which was not certified by peer review) is the author/funder. All rights reserved. No reuse allowed without permission.

47 Adams, P. D. et al. PHENIX: a comprehensive Python-based system for macromolecular structure solution. Acta Crystallogr D Biol Crystallogr 66, 213-221, doi:10.1107/s0907444909052925 (2010). 